\title{
146.
}

\section{A MEMOIR ON CURVES OF THE THIRD ORDER.}

[From the Philosophical Transactions of the Royal Society of London, vol. CXLvII. for the year 1857, pp. 415-446. Received October 30,-Read December 11, 1856.]

A CURVE of the third order, or cubic curve, is the locus represented by an equation such as $U=(*)(x, y, z)^{3}=0$; and it appears by my "Third Memoir on Quantics," [144], that it is proper to consider, in connexion with the curve of the third order $U=0$, and its Hessian $H U=0$ (which is also a curve of the third order), two curves of the third class, viz. the curves represented by the equations $P U=0$ and $Q U=0$. These equations, I say, represent curves of the third class; in fact, $P U$ and $Q U$ are contravariants of $U$, and therefore, when the variables $x, y, z$ of $U$ are considered as point coordinates, the variables $\xi, \eta, \zeta$ of $P U$ and $Q U$ must be considered as line coordinates, and the curves will be curves of the third class. I propose (in analogy with the form of the word Hessian) to call the two curves in question the Pippian and Quippian respectively. [The curve $P U=0$ is now usually called the Cayleyan.] A geometrical definition of the Pippian was readily found; the curve is in fact Steiner's curve $R_{0}$ mentioned in the memoir "Allgemeine Eigenschaften der algebraischen Curven," Crelle, t. xuviI. [1854] pp. 1-6, in the particular case of a basis-curve of the third order; and I also found that the Pippian might be considered as occurring implicitly in my "Mémoire sur les courbes du troisième ordre," Liouville, t. Ix. [1844] pp. 285-293 [26] and "Nouvelles remarques sur les courbes du troisième ordre," Liouville, t. x. [1845] pp. 102-109 [27]. As regards the Quippian, I have not succeeded in obtaining a satisfactory geometrical definition; but the search after it led to a variety of theorems, relating chiefly to the first-mentioned curve, and the results of the investigation are contained in the present memoir. Some of these resuits are due to $\mathrm{Mr}$ Salmon, with whom I was in correspondence on the subject. The character of the results makes it difficult to develope them in a systematic order; but the results are given in such connexion one with another as I have been able to present them 
in. Considering the object of the memoir to be the establishment of a distinct geometrical theory of the Pippian, the leading results will be found summed up in the nine different definitions or modes of generation of the Pippian, given in the concluding number. In the course of the memoir I give some further developments relating to the theory in the memoirs in Liouville above referred to, showing its relation to the Pippian, and the analogy with theorems of Hesse in relation to the Hessian.

\section{Article No. 1.-Definitions, \&c.}

1. It may be convenient to premise as follows:-Considering, in connexion with a curve of the third order or cubic, a point, we have:

(a) The first or conic polar of the point.

(b) The second or line polar of the point.

The meaning of these terms is well known, and they require no explanation.

Next, considering, in connexion with the cubic, a line-

(c) The first or conic polars of each point of the line meet in four points, which are the four poles of the line.

(d) The second or line polars of each point of the line envelope a conic, which is the lineo-polar envelope of the line.

And reciprocally considering, in connexion with a curve of the third class, a line, we have:

(e) The first or conic pole of the line.

(f) The second or point-pole of the line.

And considering, in connexion with the curve of the third class, a point-

(g) The first or conic poles of each line through the point touch four lines, which are the four polars of the point.

(h) The second or point poles of each line through the point generate a conic which is the point-pole locus of the point.

But I shall not have occasion in the present memoir to speak of these reciprocal figures, except indeed the first or conic pole of the line.

The term conjugate poles of a cubic is used to denote two points, such that the first or conic polar of either of them, with respect to the cubic, is a pair of lines passing through the other of them. Reciprocally, the term conjugate polars of a curve of the third class denotes two lines, such that the first or conic pole of either of them, with respect to the curve of the-third class, is a pair of points lying in the other of them. 
The expression, a syzygetic cubic, used in reference to two cubics, denotes a curve of the third order passing through the points of intersection of the two cubics; but in the present memoir the expression is in general used in reference to a single cubic, to denote a curve of the third order passing through the points of intersection of the cubic and its Hessian. As regards curves of the third class, I use in the memoir the full expression, a curve of the third class syzygetically connected with two given curves of the third class.

It is a -well-known theorem, that if at the points of intersection of a given line with a given cubic tangents are drawn to the cubic, these tangents again meet the cubic in three points which lie in a line; such line is in the present memoir termed the satellite line of the given line, and the point of intersection of the two lines is termed the satellite point of the given line; the given line in reference to its satellite line or point is termed the primary line.

In particular, if the primary line be a tangent of the cubic, the satellite line coincides with the primary line, and the satellite point is the point of simple intersection of the primary line and the cubic.

Article No. 2--Group of Theorems relating to the Conjugate Poles of a Cubic.

2. The theorems which I have first to mention relate to or criginate out of the theory of the conjugate poles of a cubic, and may be conveniently connected together and explained by means of the accompanying figure.

The point $E$ is a point of the Hessian; this being so, its first or conic polar, with respect to the cubic, will be a pair of lines passing through a point $F$ of the

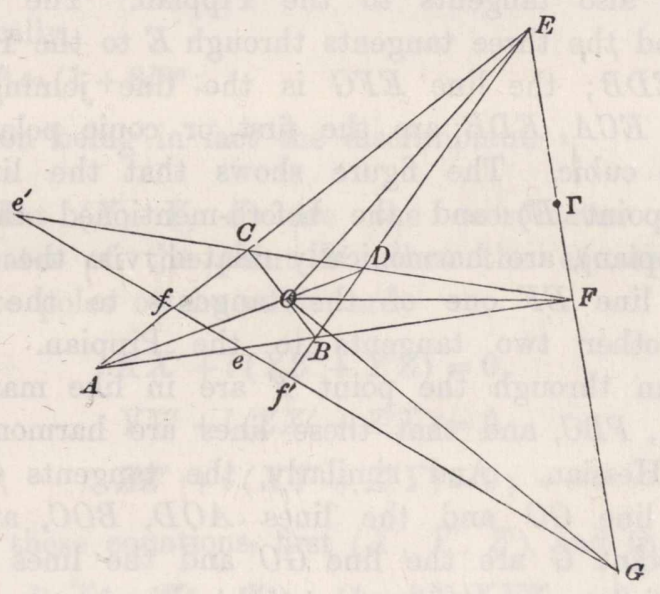

Hessian; and not only so, but the first or conic polar of the point $F$, with respect to the cubic will be a pair of lines passing through $E$. The pair of lines through 
$F$ are represented in the figure by $F B A, F D C$, and the pair of lines through $E$ are represented by $E C A, E D C$, and the lines of the one pair meet the lines of the other pair in the points $A, B, C, D$. The point $O$, which is the intersection of the lines $A D, B C$, is a point of the Hessian, and joining $E O, F O$, these lines are tangents to the Hessian at the points $E, F$, that is, the points $E, F$ are corresponding points of the Hessian, in the sense that the tangents to the Hessian at these points meet in a point of the Hessian. The two points $E, F$ are, according to a preceding definition, conjugate poles of the cubic.

The line $E F$ meets the Hessian in a third point $G$, and the points $G, O$ are conjugate poles of the cubic. The first or conic polar of $G$, with respect to the cubic, is the pair of lines $A O D, B O C$ meeting in $O$. The first or conic polar of 0 , with respect to the cubic, is the pair of lines $G E F$. and $G f^{\prime} e f e^{\prime}$ meeting in $G$. The four poles of the line $E O$, with respect to the cubic, are the points of intersection of the first or conic polars of the two points $E$ and $O$, that is, the four poles in question are the points $F, F, e, e^{\prime}$. Similarly, the four poles of the line $F O$, with respect to the cubic, are the points $E, E, f, f^{\prime}$.

The line $E F$, that is, any line joining two conjugate poles of the cubic, is a tangent to the Pippian, and the point of contact $\Gamma$ is the harmonic with respect to the points $E, F$ (which are points on the Hessian) of $G$, the third point of intersection with the Hessian. Conversely, any tangent of the Pippian meets the Hessian in three points, two of which are conjugate poles of the cubic, and the point of contact is the harmonic, with respect to these two points, of the third point of intersection with the Hessian.

The line GO in the figure is of course also a tangent of the Pippian, and moreover the lines $F B A, F D C$ (that is, the pair of-lines which are the first or conic polar of $E$ ) and the lines $E C A, E D B$ (that is, the pair of lines which are the first or conic polar of $F$ ) are also tangents to the Pippian. The point $E$ represents any point of the Hessian, and the three tangents through $E$ to the Pippian are the line $E F G$ and the lines $E C A, E D B$; the line $E F G$ is the line joining $E$ with the conjugate pole $F$, and the lines $E C A, E D B$ are the first or conic polar of this conjugate pole $F$ with respect to the cubic. The figure shows that the line $E O$ (the tangent to the Hessian at the point $E$ ) and the before-mentioned three lines (the tangents through $E$ to the Pippian), are harmonically related, viz. the line $E O$ the tangent of the Hessian, and the line $E F$ one of the tangents to the Pippian, are harmonics with respect to the other two tangents to the Pippian. It is obvious that the tangents to the Pippian through the point $F$ are in like manner the line $G F E$, and the pair of lines $F B A, F B C$, and that these lines are harmonically related to $F O$ the tangent at $F$ of the Hessian. And similarly, the tangents to the Pippian through the point $O$ are the line $G O$ and the lines $A O D, B O C$, and the tangents to the Pippian through the point $G$ are the line $G O$ and the lines $G F E$ and $G f^{\prime} e f e^{\prime}$. Thus all the lines of the figure are tangents to the Pippian except the lines EO, FO, which are tangents to the Hessian. It may be added, that the lineo-polar envelope of the line $E F$ with respect to the cubic is the pair of lines $O E, O F$. 
It will be presently seen that the analytical theory leads to the consideration of a line $I J$ (not represented in the figure): the line in question is the polar of $E$ (or $F^{\prime}$ ) with respect to the conic which is the first or conic polar of $F^{\prime}$ (or $E$ ) with respect to any syzygetic cubic. The line $I J$ is a tangent of the Pippian, and moreover the lines $E F$ and $I J$ are conjugate polars of a curve of the third class syzygetically connected with the Pippian and Quippian, and which is moreover such that its Hessian is the Pippian.

\section{Article Nos. 3 to 19.-Analytical investigations, comprising the proof of the theorems, Article No. 2.}

3. The analytical theory possesses considerable interest. Take as the equation of the cubic,

$$
U=x^{3}+y^{3}+z^{3}+6 l x y z=0 ;
$$

then the equation of the Hessian is

$$
H U=l^{2}\left(x^{3}+y^{3}+z^{3}\right)-\left(1+2 l^{3}\right) x y z=0 ;
$$

and the equation of the Pippian in line coordinates (that is, the equation which expresses that $\xi x+\eta y+\zeta z=0$ is a tangent of the curve) is

$$
P U=-l\left(\xi^{3}+\eta^{3}+\zeta^{3}\right)+\left(-1+4 l^{3}\right) \xi \eta \zeta=0 .
$$

The equation of the Quippian in line coordinates is

$$
Q U=\left(1-10 l^{3}\right)\left(\xi^{3}+\eta^{3}+\zeta^{3}\right)-6 l^{2}\left(5+4 l^{3}\right) \xi \eta \zeta=0 ;
$$

and the values of the two invariants of the cubic form are

$$
\begin{aligned}
& S=-l+l^{4}, \\
& T=1-20 l^{3}-8 l^{6},
\end{aligned}
$$

values which give identically,

$$
T^{2}-64 S^{3}=\left(1+8 l^{3}\right)^{3}
$$

the last-mentioned function being in fact the discriminant.

4. Suppose now that $(X, Y, Z)$ are the coordinates of the point $E$, and $\left(X^{\prime}, Y^{\prime}, Z^{\prime}\right)$ the coordinates of the point $F$; then the equations which express that these points are conjugate poles of the cubic, are

$$
\begin{aligned}
& X X^{\prime}+l\left(Y Z^{\prime}+Y^{\prime} Z\right)=0, \\
& Y Y^{\prime}+l\left(Z X^{\prime}+Z^{\prime} X\right)=0, \\
& Z Z^{\prime}+l\left(X Y^{\prime}+X^{\prime} Y\right)=0 ;
\end{aligned}
$$

and by eliminating from these equations, first $\left(X^{\prime}, Y^{\prime}, Z^{\prime}\right)$, and then $(X, Y, Z)$, we find

$$
\begin{aligned}
& l^{2}\left(X^{3}+Y^{3}+Z^{3}\right)-\left(1+2 l^{3}\right) X Y Z=0, \\
& l^{2}\left(X^{\prime 3}+Y^{\prime 3}+Z^{\prime 3}\right)-\left(1+2 l^{3}\right) X^{\prime} Y^{\prime} Z^{\prime}=0,
\end{aligned}
$$

which shows that the points $E$. $F$ are each of them points of the Hessian.

C. II. 
5. I may notice, in passing, that the preceding equations give rise to a somewhat singular unsymmetrical quadratic transformation of a cubic form. In fact, the second and third equations give $X^{\prime}: Y^{\prime}: Z^{\prime}=Y Z-l^{2} X^{2}: l^{2} X Y-l Z^{2}: l^{2} Z X-l Y^{2}$. And substituting these values for $X^{\prime}, Y^{\prime}, Z^{\prime}$ in the form

$$
l^{2}\left(X^{\prime 3}+Y^{\prime 3}+Z^{\prime 3}\right)-\left(1+2 l^{3}\right) X^{\prime} Y^{\prime} Z^{\prime}
$$

the result must contain as a factor

$$
l^{2}\left(X^{3}+Y^{3}+Z^{3}\right)-\left(1+2 l^{3}\right) X Y Z
$$

the other factor is easily found to be

$$
-l^{3}\left(l^{3}\left(X^{3}+Y^{3}+Z^{3}\right)+3 l X Y Z\right)
$$

Several of the formulæ given in the sequel conduct in like manner to unsymmetrical transformations of a cubic form.

6. I remark also, that the last-mentioned system of equations gives, symmetrically, $X^{\prime 2}: Y^{\prime 2}: Z^{\prime 2}: Y^{\prime} Z^{\prime}: Z^{\prime} X^{\prime}: X^{\prime} Y^{\prime}$

$$
=Y Z-l^{2} X^{2}: Z X-l^{2} Y^{2}: X Y-l^{2} Z^{2}: l^{2} Y Z-l X^{2}: l^{2} Z X-l Y^{2}: l^{2} X Y-l Z^{2} ;
$$

and it is, I think, worth showing how, by means of these relations, we pass from the equation between $X^{\prime}, Y^{\prime}, Z^{\prime}$ to that between $X, Y, Z$. In fact, representing, for shortness, the foregoing relations by

$$
X^{\prime 2}: Y^{\prime 2}: Z^{\prime 2}: Y^{\prime} Z^{\prime}: Z^{\prime} X^{\prime}: X^{\prime} Y^{\prime}=A: B: C: F: G: H,
$$

we may write

$$
X^{\prime}=A F=G H, \quad Y^{\prime}=B G=H F, \quad Z^{\prime}=C H=F G, \quad A B C=F G H ;
$$

and thence

$$
X^{\prime 3}=A F . G^{2} H^{2}, \quad Y^{\prime 3}=B G . H^{2} F^{2}, \quad Z^{\prime 3}=C H . F^{2} G^{2}, \quad X^{\prime} Y^{\prime} Z^{\prime}=F^{2} G^{2} H^{2} ;
$$

hence

$$
l^{2}\left(X^{\prime 3}+Y^{\prime 3}+Z^{\prime 3}\right)-\left(1+2 l^{3}\right) X^{\prime} Y^{\prime} Z^{\prime}=F G H\left\{l^{2}(A G H+B H F+C F G)-\left(1+2 l^{3}\right) F G H\right\} .
$$

But we have

$$
\begin{array}{rr}
l^{2}(A G H+B H F+C F G)= & -\left(2 l^{5}+l^{8}\right)\left(X^{3}+Y^{3}+Z^{3}\right) X Y Z+\left(l^{4}+2 l^{7}\right)\left(Y^{3} Z^{3}+Z^{3} X^{3}+X^{3} Y^{3}\right) \\
-\left(1+2 l^{3}\right) F G H & = \\
& \left(l^{5}+2 l^{8}\right)\left(X^{3}+Y^{3}+Z^{3}\right) X Y Z+\left(l^{4}+2 l^{7}\right)\left(Y^{3} Z^{3}+Z^{3} X^{3}+X^{3} Y^{3}\right) \\
& +l^{3}\left(1-l^{3}\right)\left(1+2 l^{3}\right) X^{2} Y^{2} Z^{2}
\end{array}
$$

and thence

$$
\begin{aligned}
l^{2}(A G H+B H F+C F G)- & \left(1+2 l^{3}\right) F G H \\
& =-l^{3}\left(1-l^{3}\right)\left\{l^{2}\left(X^{3}+Y^{3}+Z^{3}\right) X Y Z-\left(1+2 l^{3}\right) X^{2} Y^{2} Z^{2}\right\}
\end{aligned}
$$

and finally,

$$
\begin{array}{r}
l^{2}\left(X^{\prime 3}+Y^{\prime 3}+Z^{\prime 3}\right)-\left(1+2 l^{3}\right) X^{\prime} Y^{\prime} Z^{\prime}=l^{5}\left(-l+l^{4}\right)\left(l Y Z-X^{2}\right)\left(l Z X-Y^{2}\right)\left(l X Y-Z^{2}\right) X Y Z \\
\times\left\{l^{2}\left(X^{3}+Y^{3}+Z^{3}\right)-\left(1+2 l^{3}\right) X Y Z\right\}
\end{array}
$$


We have also, identically,

$$
A B C-F G H=\frac{1}{l}\left(-l+l^{4}\right) X Y Z\left\{l^{2}\left(X^{3}+Y^{3}+Z^{3}\right)-\left(1+2 l^{3}\right) X Y Z\right\},
$$

which agrees with the relation $A B C-F G H=0$.

7. Before going further, it will be convenient to investigate certain relations which exist between the quantities $(X, Y, Z),\left(X^{\prime}, Y^{\prime}, Z^{\prime}\right)$, connected as before by the equations

$$
\begin{aligned}
& X X^{\prime}+l\left(Y Z^{\prime}+Y^{\prime} Z\right)=0 \\
& Y Y^{\prime}+l\left(Z X^{\prime}+Z^{\prime} X\right)=0 \\
& Z Z^{\prime}+l\left(X Y^{\prime}+X^{\prime} Y\right)=0
\end{aligned}
$$

and the quantities

$$
\begin{array}{ll}
\xi=Y Z^{\prime}-Y^{\prime} Z, & \alpha=X X^{\prime}=-\frac{1}{l}\left(Y Z^{\prime}+Y^{\prime} Z\right), \\
\eta=Z X^{\prime}-Z^{\prime} X, & \beta=Y Y^{\prime}=-\frac{1}{l}\left(Z X^{\prime}+Z^{\prime} X\right), \\
\zeta=X Y^{\prime}-X^{\prime} Y, & \gamma=Z Z^{\prime}=-\frac{1}{l}\left(X Y^{\prime}+X^{\prime} Y\right) .
\end{array}
$$

We have identically,

$$
2 X X^{\prime}\left(Y Z^{\prime}-Y^{\prime} Z\right)+\left(X Y^{\prime}+X^{\prime} Y\right)\left(Z X^{\prime}-Z^{\prime} X\right)+\left(Z X^{\prime}+Z^{\prime} X\right)\left(X Y^{\prime}-X^{\prime} Y\right)=0 ;
$$

or expressing in terms of $\xi, \eta, \zeta, \alpha, \beta, \gamma$ the quantities which enter into this equation, and forming the analogous equations, we have

$$
\begin{aligned}
& 2 l \alpha \xi-\gamma \eta-\beta \zeta=0 \\
& -\gamma \xi+2 l \beta \eta-\alpha \zeta=0 \\
& -\beta \xi-\quad \alpha \eta+2 l y \zeta=0 .
\end{aligned}
$$

We have also

$$
X^{2} Y^{\prime} Z^{\prime}-X^{\prime 2} Y Z=\frac{1}{2}\left\{-\left(X Y^{\prime}+X^{\prime} Y\right)\left(Z X^{\prime}-Z^{\prime} X\right)+\left(Z X^{\prime}+Z^{\prime} X\right)\left(X Y^{\prime}-X^{\prime} Y\right)\right\},
$$

and thence in like manner,

$$
\begin{aligned}
& X^{2} Y^{\prime} Z^{\prime}-X^{\prime 2} Y Z=\frac{1}{2 l}(\gamma \eta-\beta \zeta) \\
& Y^{2} Z^{\prime} X^{\prime}-Y^{\prime 2} Z X=\frac{1}{2 l}(\alpha \zeta-\gamma \xi) \\
& Z^{2} X^{\prime} Y^{\prime}-X^{\prime 2} Y Z=\frac{1}{2 l}(\beta \xi-\alpha \eta)
\end{aligned}
$$

Again, we have

$$
\begin{aligned}
& \left(Y Z^{\prime}-Y^{\prime} Z\right)^{2}=\left(Y Z^{\prime}+Y^{\prime} Z\right)^{2}-4 Y Y^{\prime} Z Z^{\prime} \\
& \left(Z X^{\prime}-Z^{\prime} X\right)\left(X Y^{\prime}-X^{\prime} Y\right)=-\left(Z X^{\prime}+Z^{\prime} X\right)\left(X Y^{\prime}+X^{\prime} Y\right)+2 X X^{\prime}\left(Y Z^{\prime}+Y^{\prime} Z\right)
\end{aligned}
$$


and thence

$$
\begin{aligned}
& \xi^{2}=\frac{1}{l^{2}} \alpha^{2}-4 \beta \gamma \\
& \eta^{2}=\frac{1}{l^{2}} \beta^{2}-4 \gamma \alpha \\
& \zeta^{2}=\frac{1}{l^{2}} \gamma^{2}-4 \alpha \beta \\
& \eta \zeta=-\frac{2}{l} \alpha^{2}-\frac{1}{l^{2}} \beta \gamma \\
& \zeta \xi=-\frac{2}{l} \beta^{2}-\frac{1}{l^{2}} \gamma \alpha \\
& \xi \eta=-\frac{2}{l} \gamma^{2}-\frac{1}{l^{2}} \alpha \beta
\end{aligned}
$$

and conversely

$$
\begin{aligned}
\frac{1}{l^{2}}\left(1+8 l^{3}\right) \alpha^{2} & =\xi^{2}-4 l^{2} \eta \zeta, \\
\frac{1}{l^{2}}\left(1+8 l^{3}\right) \beta^{2} & =\eta^{2}-4 l^{2} \zeta \xi, \\
\frac{1}{l^{2}}\left(1+8 l^{3}\right) \gamma^{2} & =\zeta^{2}-4 l^{2} \xi \eta, \\
-\frac{1}{l^{2}}\left(1+8 l^{3}\right) \beta \gamma & =2 l \xi^{2}+\eta \zeta, \\
-\frac{1}{l^{2}}\left(1+8 l^{3}\right) \gamma \alpha & =2 l \eta^{2}+\zeta \xi, \\
-\frac{1}{l^{2}}\left(1+8 l^{3}\right) \alpha \beta & =2 l \zeta^{2}+\xi \eta .
\end{aligned}
$$

8. It is obvious that

$$
\xi x+\eta y+\zeta z=0
$$

is the equation of the line $E F$ joining the two conjugate poles, and it may be shown that

$$
\alpha x+\beta y+\gamma z=0
$$

is the equation of the line $I J$, which is the polar of $E$ with respect to a conic which is the first or conic polar of $F$ with respect to any syzygetic cubic. In fact, the equation of a syzygetic cubic will be $x^{3}+y^{3}+z^{3}+6 \lambda x y z=0$, where $\lambda$ is arbitrary, and the equation of the line in question is

$$
\left(X \partial_{x}+Y \partial_{y}+Z \partial_{z}\right)\left(X^{\prime} \partial_{x}+Y^{\prime} \partial_{y}+Z^{\prime} \partial_{z}\right)\left(x^{3}+y^{3}+2^{3}+6 \lambda x y z\right)=0
$$

or developing,

$$
\begin{aligned}
& X X^{\prime} x+Y Y^{\prime} y+Z Z^{\prime} z \\
& \left.+\lambda\left\{Y Z^{\prime}+Y^{\prime} Z\right) x+\left(Z X^{\prime}+Z^{\prime} X\right) y+\left(X Y^{\prime}+X^{\prime} Y\right) z\right\}=0
\end{aligned}
$$


and the function on the left-hand side is

$$
\left(1-\frac{\lambda}{l}\right)(\alpha x+\beta y+\gamma z)
$$

which proves the theorem.

9. The equations (A) by the elimination of $(\xi, \eta, \zeta)$, give

$$
-l\left(\alpha^{3}+\beta^{3}+\gamma^{3}\right)+\left(-1+4 l^{3}\right) \alpha \beta \gamma=0,
$$

which shows that the line $I J$ is a tangent of the Pippian: the proof of the theorem is given in this place because the relation just obtained between $\alpha, \beta, \gamma$ is required for the proof of some of the other theorems.

10. To find the coordinates of the point $G$ in which the line $E F$ joining two conjugate poles again meets the Hessian.

We may take for the coordinates of $G$,

$$
u X+v X^{\prime \prime}, \quad u Y+v Y^{\prime}, \quad u Z+v Z^{\prime}
$$

and, substituting in the equation of the Hessian, the terms containing $u^{3}, v^{3}$ disappear, and the ratio $u: v$ is determined by a simple equation. It thus appears that we may write

$$
\begin{aligned}
& u=-3 l^{2}\left(X X^{\prime 2}+Y Y^{\prime 2}+Z Z^{\prime 2}\right)+\left(1+2 l^{3}\right)\left(Y^{\prime} Z^{\prime} X+Z^{\prime} X^{\prime} Y+X^{\prime} Y^{\prime} Z\right), \\
& v=3 l^{2}\left(X^{2} X^{\prime}+Y^{2} Y^{\prime}+Z^{2} Z^{\prime}\right)-\left(1+2 l^{3}\right)\left(Y Z X^{\prime}+Z X Y^{\prime}+X Y Z^{\prime}\right) ;
\end{aligned}
$$

hence introducing, as before, the quantities $\xi, \eta, \zeta, \alpha, \beta, \gamma$, we find

$$
u X+v X^{\prime}=3 l^{2}(\gamma \eta-\beta \zeta)+\left(1+2 l^{3}\right)\left(X^{2} Y^{\prime} Z^{\prime}-X^{\prime 2} Y Z\right)
$$

but from the first of the equations (B),

$$
X^{2} Y^{\prime} Z^{\prime}-X^{\prime 2} Y Z=\frac{1}{2 l}(\gamma \eta-\beta \zeta)
$$

and therefore the preceding value of $u X+v X^{\prime}$ becomes

$$
\left(3 l^{2}-\frac{1+2 l^{3}}{2 l}\right)(\gamma \eta-\beta \zeta)
$$

which is equal to

$$
\frac{-1+4 l^{3}}{2 l}(\gamma \eta-\beta \zeta)
$$

Hence throwing out the constant factor, we find, for the coordinates of the point $G$, the values

$$
\gamma \eta-\beta \zeta, \quad \alpha \zeta-\gamma \xi, \quad \beta \xi-\alpha \eta .
$$

11. To find the coordinates of the point 0 .

Consider $O$ as the point of intersection of the tangents to the Hessian at the points $E, F$, then the coordinates of $O$ are proportional to the terms of

$$
\left\|\begin{array}{lll}
3 l^{2} X^{2}-\overline{1+2 l^{3}} Y Z, & 3 l^{2} Y^{2}-\overline{1+2 l^{3}} Z X, & 3 l^{2} Z^{2}-\overline{1+2 l^{3}} X Y \\
3 l^{2} X^{\prime 2}-\overline{1+2 l^{3}} Y^{\prime} Z^{\prime}, & 3 l^{2} Y^{\prime 2}-\overline{1+2 l^{3}} Z^{\prime} X^{\prime}, & 3 l^{2} Z^{\prime 2}-\overline{1+2 l^{3}} X^{\prime} Y^{\prime}
\end{array}\right\|
$$


Hence the $x$-coordinate is proportional to

$$
\left.\left(3 l^{2} Y^{2}-\overline{1+2 l^{3}} Z X\right)\left(3 l^{2} Z^{\prime 2}-\overline{1+2 l^{3}} X^{\prime} Y^{\prime}\right)-\left(3 l^{2} Z^{2}-\overline{1+2 l^{3}} X Y\right) 3 l^{2} Y^{\prime 2}-\overline{1+2 l^{3}} Z^{\prime} X^{\prime}\right),
$$
which is equal to

$$
\begin{array}{r}
9 l^{4}\left(Y^{2} Z^{\prime 2}-Y^{\prime 2} Z^{2}\right)+3 l^{2}\left(1+2 l^{3}\right) Y Y^{\prime}\left(X Y^{\prime}-X^{\prime} Y\right)+3 l^{2}\left(1+2 l^{3}\right) Z Z^{\prime}\left(Z X^{\prime}-Z^{\prime} X\right) \\
-\left(1+2 l^{3}\right)^{2} X X^{\prime}\left(Y Z^{\prime}-Y^{\prime} Z\right) ;
\end{array}
$$

or introducing, as before, the quantities $\xi, \eta, \zeta, \alpha, \beta, \gamma$, to

$$
\begin{aligned}
& -9 l^{3} \alpha \xi+3 l^{2}\left(1+2 l^{3}\right)(\beta \zeta+\gamma \eta)-\left(1+2 l^{3}\right)^{2} \alpha \xi, \\
& =\left(-1-13 l^{3}-4 l^{6}\right) \alpha \xi+2 l^{2}\left(1+2 l^{3}\right)(\beta \zeta+\gamma \eta) .
\end{aligned}
$$

But by the first of the equations (A) $\beta \zeta+\gamma \eta=2 l \alpha \xi$, and the preceding value thus becomes $\left(-1-7 l^{3}+8 l^{6}\right) \alpha \xi$. Hence throwing out the constant factor the coordinates of the point $O$ are found to be

$$
\alpha \xi, \quad \beta \eta, \gamma \zeta .
$$

12. The points $G, O$ are conjugate poles of the cubic.

Take $a, b, c$ for the coordinates of $G$, and $a^{\prime}, b^{\prime}, c^{\prime}$ for the coordinates of $O$, we have

$$
\begin{aligned}
& a, b, c=\gamma \eta-\beta \zeta, \quad \alpha \zeta-\gamma \xi, \quad \beta \xi-\alpha \eta, \\
& a^{\prime}, b^{\prime}, c^{\prime}=\alpha \xi \quad, \beta \eta \quad, \gamma \zeta .
\end{aligned}
$$

These values give $a a^{\prime}+l\left(b c^{\prime}+b^{\prime} c\right)$

$$
\begin{aligned}
& =\alpha \xi(\gamma \eta-\beta \zeta)+l\{\beta \eta(\beta \xi-\alpha \eta)+\gamma \zeta(\alpha \zeta-\gamma \xi)\} \\
& =\xi \eta\left(\alpha \gamma+l \beta^{2}\right)+\eta^{2}(-l \alpha \beta)+\zeta^{2}(l \alpha \gamma)+\xi \zeta\left(-\alpha \beta-l \gamma^{2}\right) ;
\end{aligned}
$$

or substituting for $\xi \eta, \eta^{2}, \zeta^{2}, \xi \xi$ their values in terms of $\alpha, \beta, \gamma$, this is

$$
\begin{aligned}
& \left(-\frac{2}{l} \gamma^{2}-\frac{1}{l^{2}} \alpha \beta\right)\left(\alpha \gamma+l \beta^{2}\right) \\
+ & \left(\frac{\beta^{2}}{l^{2}}-4 \gamma \alpha\right)(-l \alpha \beta) \\
+ & \left(\frac{\gamma^{2}}{l^{2}}-4 \alpha \beta\right)(\quad l \alpha \gamma) \\
+ & \left(-\frac{2}{l} \beta^{2}-\frac{1}{l} \alpha \gamma\right)\left(-\alpha \beta-l \gamma^{2}\right),
\end{aligned}
$$

which is identically equal to zero. Hence, completing the system, we find

$$
\begin{aligned}
& a a^{\prime}+l\left(b c^{\prime}+b^{\prime} c\right)=0, \\
& b b^{\prime}+l\left(c a^{\prime}+c^{\prime} a\right)=0, \\
& c c^{\prime}+l\left(a b^{\prime}+a^{\prime} b\right)=0,
\end{aligned}
$$

equations which show that $O$ (as well as $G$ ) is a point of the Hessian, and that the points $G, O$ are corresponding poles of the cubic. 
13. The line $E F$ joining a pair of conjugate poles of the cubic is a tangent of the Pippian'.

In fact, the equations (A), by the elimination of $\alpha, \beta, \gamma$, give

$$
-l\left(\xi^{3}+\eta^{3}+\zeta^{3}\right)+\left(-1+4 l^{3}\right) \xi \eta \zeta=0,
$$

which proves the theorem.

14. To find the equation of the pair of lines through $F$, and to show that these lines are tangents of the Pippian.

The equation of the pair of lines considered as the first or conic polar of the conjugate pole $E$, is

$$
X\left(x^{2}+2 l y z\right)+Y\left(y^{2}+2 l z x\right)+Z\left(z^{2}+2 l x y\right)=0 .
$$

Let one of the lines be

then the other is

$$
\lambda x+\mu y+\nu z=0
$$

$$
\frac{X}{\lambda} x+\frac{Y}{\mu} y+\frac{Z}{\nu} z=0
$$

and we find

$$
\begin{array}{r}
2 l X \mu \nu-Y \nu^{2}-Z \mu^{2}=0 \\
-X \nu^{2}+2 l Y \nu \lambda-Z \lambda^{2}=0, \\
-X \mu^{2}-Y \lambda^{2}+2 l Z \mu \nu=0,
\end{array}
$$

any two of which determine the ratios $\lambda, \mu, \nu$.

The elimination of $X, Y, Z$ gives

which is equivalent to

$$
\left|\begin{array}{ccc}
2 l \mu \nu, & -\nu^{2}, & -\mu^{2} \\
-\nu^{2}, & 2 l \nu \lambda, & -\lambda^{2} \\
-\mu^{2}, & -\lambda^{2}, & 2 l \lambda \mu
\end{array}\right|=0,
$$

$$
\lambda \mu \nu\left\{-l\left(\lambda^{3}+\mu^{3}+\nu^{3}\right)+\left(-1+4 l^{3}\right) \lambda \mu \nu\right\}=0 ;
$$

or, omitting a factor, to

$$
-l\left(\lambda^{3}+\mu^{3}+\nu^{3}\right)+\left(-1+4 l^{3}\right) \lambda \mu \nu=0,
$$

which shows that the line in question is a tangent of the Pippian.

15. To find the equation of the pair of lines through 0 .

The equation of the pair of lines through $E$ is in like manner

$$
X^{\prime}\left(x^{2}+2 l y z\right)+Y^{\prime}\left(y^{2}+2 l z x\right)+Z^{\prime}\left(z^{2}+2 l x y\right)=0 ;
$$

${ }^{1}$ Steiner's curve $R_{0}$, in the particular case of a cubic basis-curve, is according to definition the envelope of the line $E F$, that is, the curve $R_{0}$ in the particular case in question is the Pippian. 
and combining this with the foregoing equation,

$$
X\left(x^{2}+2 l y z\right)+Y\left(y^{2}+2 l z x\right)+Z\left(z^{2}+2 l x y\right)=0
$$

of the pair of lines through $F$, viz. multiplying the two equations by

$$
X^{2} X^{\prime}+Y^{2} Y^{\prime}+Z^{2} Z^{\prime}, \quad-\left(X X^{\prime 2}+Y Y^{\prime 2}+Z Z^{\prime 2}\right),
$$

and adding, then if as before

$$
a: b: c=\gamma \eta-\beta \zeta: \alpha \zeta-\gamma \xi: \beta \xi-\alpha \eta,
$$

we find as the equation of a conic passing through the points $A, B, C, D$, the equation

$$
a\left(x^{2}+2 l y z\right)+b\left(y^{2}+2 l z x\right)+c\left(z^{2}+2 l x y\right)=0 .
$$

But putting, as before,

$$
a^{\prime}: b^{\prime}: c^{\prime}=\alpha \xi: \beta \eta: \gamma \zeta,
$$

then $a^{\prime}, b^{\prime}, c^{\prime}$ are the coordinates of the point $O$, and the equations

$$
\begin{aligned}
& a a^{\prime}+l\left(b c^{\prime}+b^{\prime} c\right)=0, \\
& b b^{\prime}+l\left(c a^{\prime}+c^{\prime} a\right)=0, \\
& c c^{\prime}+l\left(a b^{\prime}+a^{\prime} b\right)=0,
\end{aligned}
$$

show that the conic in question is in fact the pair of lines through the point $O$.

16. To find the coordinates of the point $\Gamma$, which is the harmonic of $G$ with respect to the points $E, F$.

The coordinates of the point in question are

$$
u X-v X^{\prime}, \quad u Y-v Y^{\prime}, \quad u Z-v Z^{\prime},
$$

where $u, v$ have the values given in No. 10, viz.

$$
\begin{aligned}
& u=-3 l^{2}\left(X X^{\prime 2}+Y Y^{\prime 2}+Z Z^{\prime 2}\right)+\left(1+2 l^{3}\right)\left(Y^{\prime} Z^{\prime} X+Z^{\prime} X^{\prime} Y+X^{\prime} Y^{\prime} Z\right), \\
& v=3 l^{2}\left(X^{2} X^{\prime}+Y^{2} Y^{\prime}+Z^{2} Z^{\prime}\right)-\left(1+2 l^{3}\right)\left(Y Z X^{\prime}+Z X Y^{\prime}+X Y Z^{\prime}\right) ;
\end{aligned}
$$

these values give

$$
\begin{aligned}
u X-v X^{\prime}=-3 l^{2}\left\{2 X^{2} X^{\prime 2}\right. & \left.+\left(X Y^{\prime}+X^{\prime} Y\right) Y Y^{\prime}+\left(X Z^{\prime}+X^{\prime} Z\right) Z Z^{\prime}\right\} \\
& +\left(1+2 l^{3}\right)\left\{\left(X Y^{\prime}+X^{\prime} Y\right)\left(X Z^{\prime}+X^{\prime} Z\right)+X X^{\prime}\left(Y Z^{\prime}+Y^{\prime} Z\right\}\right.
\end{aligned}
$$

and therefore

$$
\begin{aligned}
u X-v X^{\prime} & =-3 l^{2}\left\{2 \alpha^{2}-\frac{2}{l} \beta \gamma\right\}+\left(1+2 l^{3}\right)\left\{\frac{1}{l^{2}} \beta \gamma-\frac{1}{l} \alpha^{2}\right\} \\
& =\frac{1}{l^{2}}\left(1+8 l^{3}\right)\left(-l \alpha^{2}+\beta \gamma\right)
\end{aligned}
$$

and consequently, omitting the constant factor, the coordinates of $\Gamma$ may be taken to be

$$
-l \alpha^{2}+\beta \gamma, \quad-l \beta^{2}+\gamma \alpha, \quad-l \gamma^{2}+\alpha \beta .
$$


17. The line through two consecutive positions of the point $\Gamma$ is the line $E F$.

The coordinates of the point $\Gamma$ are

$$
-l \alpha^{2}+\beta \gamma, \quad-l \beta^{2}+\gamma \alpha, \quad-l \gamma^{2}+\alpha \beta
$$

and it has been shown that the quantities $\alpha, \beta, \gamma$ satisfy the equation

$$
-l\left(a^{3}+\beta^{3}+\gamma^{3}\right)+\left(-1+4 l^{3}\right) \alpha \beta \gamma=0 .
$$

Hence, considering $\alpha, \beta, \gamma$ as variable parameters connected by this equation, the equation of the line through two consecutive positions of the point $\Gamma$ is

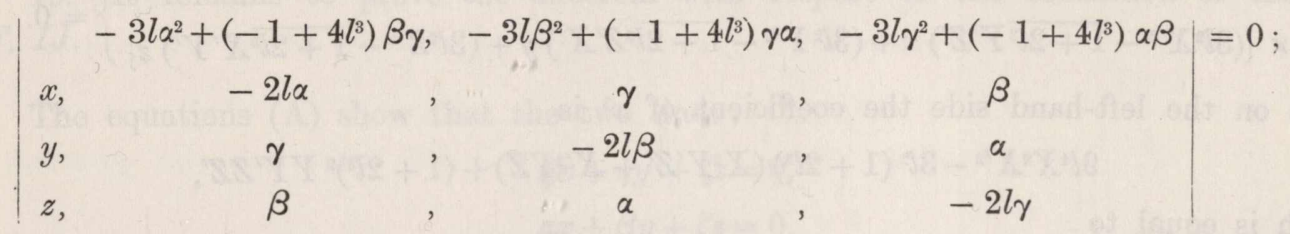

and representing this equation by

$$
L x+M y+N z=0,
$$

we find

$$
\begin{aligned}
L= & \left(4 l^{2} \beta \gamma-\alpha^{2}\right)\left(-3 l \alpha^{2}+\left(-1+4 l^{3}\right) \beta \gamma\right) \\
& +\left(\alpha \beta+2 l \gamma^{2}\right)\left(-3 l \beta^{2}+\left(-1+4 l^{3}\right) \gamma \alpha\right) \\
& +\left(\alpha \gamma+2 l \beta^{2}\right)\left(-3 l \gamma^{2}+\left(-1+4 l^{3}\right) \alpha \beta\right)
\end{aligned}
$$

or, multiplying out and collecting,

$$
L=3 l \alpha^{4}+\left(-1-8 l^{3}\right) \alpha^{2} \beta \gamma+\left(-5 l+8 l^{4}\right)\left(\alpha \beta^{3}+\alpha \gamma^{3}\right)+\left(-16 l^{2}+16 l^{5}\right) \beta^{2} \gamma^{2} ;
$$

but the equation

$$
-l\left(\alpha^{3}+\beta^{3}+\gamma^{3}\right)+\left(-1+4 l^{3}\right) \alpha \beta \gamma=0
$$

gives

$$
3 l \alpha^{4}=-3 l\left(\alpha \beta^{3}+\alpha \gamma^{3}\right)+\left(-3+12 l^{3}\right) \alpha^{2} \beta \gamma
$$

and we have

$$
\begin{aligned}
L & =\left(-4+4 l^{3}\right) \alpha^{2} \beta \gamma+\left(-8 l+8 l^{4}\right)\left(\alpha \beta^{3}+\alpha \gamma^{3}\right)+\left(-16 l^{2}+16 l^{5}\right) \beta^{2} \gamma^{2} \\
& =\left(-4+4 l^{3}\right)\left(\alpha^{2} \beta \gamma+2 l\left(\alpha \beta^{3}+\alpha \gamma^{3}\right)+4 l^{2} \beta^{2} \gamma^{2}\right) \\
& =\left(-4+4 l^{3}\right)\left(\alpha \gamma+2 l \beta^{2}\right)\left(\alpha \beta+2 l \gamma^{2}\right)
\end{aligned}
$$

or, in virtue of the equations (D),

$$
L=\left(-4+4 l^{3}\right) l^{2} \zeta \xi . l^{2} \xi \eta=\left(-4+4 l^{3}\right) l^{4} \xi^{2} \eta \zeta=\left(-4+4 l^{3}\right) l^{4} \xi \eta \zeta . \xi .
$$

Hence, omitting the common factor, we find $L: M: N=\xi: \eta: \zeta$, and the equation $L x+M y+N z=0$ becomes

$$
\xi x+\eta y+\zeta z=0,
$$

C. II. 
which is the equation of the line $E F$, that is, the line through two consecutive positions of $\Gamma$ is the line $E F$; or what is the same thing, the line $E F$ touches the Pippian in the point $\Gamma$ which is the harmonic of $G$ with respect to the points $E, F$.

18. The lineo-polar envelope of the line $E F$, with respect to the cubic, is the pair of lines $O E, O F$.

The equation of the pair of iines $O E, O F$, considered as the tangents to the Hessian at the points $E, F$, is

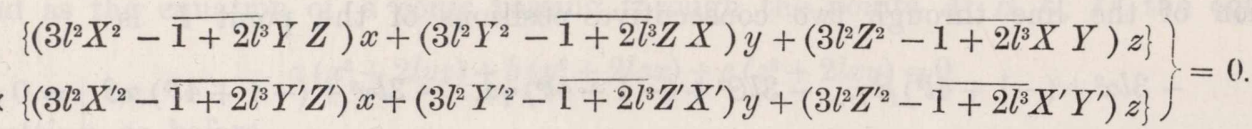

Here on the left-hand side the coefficient of $x^{2}$ is

$$
9 l^{4} X^{2} X^{\prime 2}-3 l^{2}\left(1+2 l^{3}\right)\left(X^{2} Y^{\prime} Z^{\prime}+X^{\prime 2} Y Z\right)+\left(1+2 l^{3}\right)^{2} Y Y^{\prime} Z Z^{\prime},
$$

which is equal to

$$
9 l^{4} \alpha^{2}-3 l^{2}\left(1+2 l^{3}\right)\left(l^{2} \beta \gamma+\frac{1}{l} \alpha^{2}\right)+\left(1+2 l^{3}\right)^{2} \beta \gamma
$$

that is

$$
\frac{1}{l}\left(-l+l^{4}\right)\left\{3 l \alpha^{2}+2\left(1+2 l^{3}\right) \beta \gamma\right\}
$$

and the coefficient of $y z$ is

$$
\begin{aligned}
9 l^{4}\left(Y^{2} Z^{\prime 2}+Y^{\prime 2} Z^{2}\right)-3 l^{2}\left(1+2 l^{3}\right)\left(Y Y^{\prime}\left(X Y^{\prime}+X^{\prime} Y\right)+Z Z^{\prime}\left(X Z^{\prime}+X^{\prime} Z\right)\right) \\
+\left(1+2 l^{3}\right)^{2} X X^{\prime}\left(Y Z^{\prime}+Y^{\prime} Z\right),
\end{aligned}
$$

which is equal to

that is

$$
9 l^{4}\left(\frac{1}{l^{2}} \alpha^{2}-2 \beta \gamma\right)-3 l^{2}\left(1+2 l^{3}\right)\left(-\frac{2}{l} \beta \gamma\right)+\left(1+2 l^{3}\right)^{2} \alpha\left(-\frac{1}{l} \alpha\right),
$$

$$
\frac{1}{l}\left(-l+l^{4}\right)\left\{\left(1-4 l^{3}\right) a^{2}-6 l^{2} \beta \gamma\right\} .
$$

Hence completing the system and throwing out the constant factor, the equation of the pair of lines is

$$
\begin{aligned}
& \left(3 l \alpha^{2}+2\left(1+2 l^{3}\right) \beta \gamma, \quad 3 l \beta^{2}+2\left(1+2 l^{3}\right) \gamma \alpha, \quad 3 l \gamma^{2}+2\left(1+2 l^{3}\right) \alpha \beta,\right. \\
& \left.\left(1-4 l^{3}\right) \alpha^{2}-6 l^{2} \beta \gamma, \quad\left(1-4 l^{3}\right) \beta^{2}-6 l^{2} \gamma \alpha, \quad\left(1-4 l^{3}\right) \gamma^{2}-6 l^{2} \alpha \beta^{\top} \gamma x, y, z\right)^{2}=0 .
\end{aligned}
$$

But the equation of the line $E F$ is $\xi x+\eta y+\zeta z=0$, and the equation of its lineo-polar envelope is

$$
\begin{array}{llll} 
& \xi, & \eta, & \zeta \\
\xi, & x, & l z, & l y \\
\eta, & l z, & y, & l x \\
\zeta, & l y, & x, & z
\end{array} \mid=0 ;
$$


or expanding,

$$
\left(y z-l^{2} x^{2}, \quad z x-l^{2} y^{2}, \quad x y-l^{2} z^{2}, \quad l^{2} y z-l x^{2}, \quad l^{2} z x-l y^{2}, \quad l^{2} x y-l z^{2} \gamma \xi, \quad \eta, \quad \zeta\right)^{2}=0 ;
$$

or arranging in powers of $x, y, z$,

$$
\left(-l^{2} \xi^{2}-2 l \eta \zeta, \quad-l^{2} \eta^{2}-2 l \zeta \xi, \quad-l^{2} \zeta^{2}-2 l \xi \eta, \quad \frac{1}{2} \xi^{2}+l^{2} \eta \zeta, \quad \frac{1}{2} \eta^{2}+l^{2} \zeta \xi, \quad \frac{1}{2} \zeta^{2}+l^{2} \xi \eta \gamma x, y, z\right)^{2}=0:
$$

and if in this equation we replace $\xi^{2}$, \&c. by their values in terms of $\alpha, \beta, \gamma$, as given by the equations (D), we obtain the equation given as that of the pair of lines $O E, O F$.

19. It remains to prove the theorem with respect to the connexion of the lines $E F, I J$.

The equations (A) show that the two lines

$$
\begin{aligned}
& \xi x+\eta y+\zeta z=0, \\
& \alpha x+\beta y+\zeta z=0,
\end{aligned}
$$

(where $\xi, \eta, \zeta$ and $\alpha, \beta, \gamma$ have the values before attributed to them) are conjugate polars with respect to the curve of the third class,

$$
l\left(\xi^{3}+\eta^{3}+\zeta^{3}\right)-3 \xi \eta \zeta=0,
$$

in which equation $\xi, \eta, \zeta$ denote current line coordinates. The curve in question is of the form $A P U+B Q U=0$. We have, in fact, identically,

$$
3 T \cdot P U-4 S \cdot Q U=\left(1+8 l^{3}\right)^{2}\left\{l\left(\xi^{3}+\eta^{3}+\zeta^{3}\right)-3 \xi \eta \zeta\right\} .
$$

It is clear that the curve in question must have the curve $P U=0$ for its Hessian; and in fact, in the formula of my Third Memoir, [144]

$$
\begin{aligned}
H(6 \alpha P U+\beta Q U) & =\left(-2 T, 48 S^{2}, 18 T S, T^{2}+16 S^{3} \gamma(\alpha, \beta)^{3}, P U\right. \\
& +\left(8 S, \quad T,-8 S^{2}, \quad-T S \quad \gamma \alpha, \beta\right)^{3} Q U,
\end{aligned}
$$

the coefficient of $Q U$ is

$$
(8 S \alpha+T \beta)\left(\alpha^{2}-S \beta^{2}\right)
$$

and therefore, putting $\alpha=\frac{1}{2} T, \beta=-4 S$, we find

$$
H(3 T . P U-4 S . Q U)=-\frac{1}{4}\left(T^{2}-64 S^{3}\right)^{2} P U .
$$

Article No. 20.-Theorem relating to the curve of the third class, mentioned in the preceding Article.

20. The consideration of the curve $3 T \cdot P U-4 S \cdot Q U=0$, gives rise to another geometrical theorem. Suppose that the line $(\xi, \eta, \xi)$, that is, the line whose equation is $\xi x+\eta y+\xi z=0$, is with respect to this curve of the third class one of the four polars of a point $(X, Y, Z)$ of the Hessian, and that it is required to find the envelope of the line $\xi x+\eta y+\zeta z=0$. 
We have

$$
X: Y: Z=l \xi^{2}-\eta \zeta: l \eta^{2}-\zeta \xi: l \zeta^{2}-\xi \eta,
$$

and $X, Y, Z$ are to be eliminated from these equations, and the equation

$$
l^{2}\left(X^{3}+Y^{3}+Z^{3}\right)-\left(1+2 l^{3}\right) X Y Z=0
$$

of the Hessian. We have

$$
\begin{aligned}
X^{3}+Y^{3}+Z^{3}= & l^{3}\left(\xi^{3}+\eta^{3}+\zeta^{3}\right)^{2} \\
& -3 l^{2}\left(\xi^{3}+\eta^{3}+\zeta^{3}\right) \xi \eta \zeta \\
& +9 l \xi^{2} \eta^{2} \zeta^{2} \\
& -\left(1+2 l^{3}\right)\left(\eta^{3} \zeta^{3}+\zeta^{3} \xi^{3}+\xi^{3} \eta^{3}\right) \\
X Y Z= & l\left(\xi^{3}+\eta^{3}+\zeta^{3}\right) \xi \eta \zeta \\
& +\left(-1+l^{3}\right) \xi^{2} \eta^{2} \zeta^{2} \\
& -l^{2}\left(\eta^{3} \zeta^{3}+\zeta^{3} \xi^{3}+\xi^{3} \eta^{3}\right)
\end{aligned}
$$

and thence

$$
\begin{aligned}
H U= & l^{5}\left(\xi^{3}+\eta^{3}+\zeta^{3}\right)^{2} \\
& -\left(l+5 l^{4}\right)\left(\xi^{3}+\eta^{3}+\zeta^{3}\right) \xi \eta \zeta \\
& +\left(1+10 l^{3}-2 l^{6}\right) \xi^{2} \eta^{2} \zeta^{2}
\end{aligned}
$$

and equating the right-hand side to zero, we have the equation in line coordinates of the curve in question, which is therefore a curve of the sixth class in quadratic syzygy with the Pippian and Quippian.

\section{Article No. 21.-Geometrical definition of the Quippian.}

21. I have not succeeded in obtaining any good geometrical definition of the Quippian, and the following is only given for want of something better.

The curve

$$
T . P U\{P 6 H(\alpha U+6 \beta H U)\}-P(6 H U)\{T(\alpha U+6 \beta H U) \cdot P(\alpha U+6 \beta H U)\}=0,
$$

which is derived in what may be taken to be a known manner from the cubic, is in general a curve of the sixth class. But if the syzygetic cubic $\alpha U+6 \beta H U=0$ be properly selected, viz. if this curve be such that its Hessian breaks up into three lines, then both the Pippian of the cubic $\alpha U+6 \beta H U=0$, and the Pippian of its Hessian will break up into the same three points, which will be a portion of the curve of the sixth class, and discarding these three points the curve will sink down to one of the third class, and will in fact be the Quippian of the cubic.

To show this we may take

$$
\alpha U+6 \beta H U=x^{3}+y^{3}+z^{3},=0
$$


as the equation of the syzygetic cubic satisfying the prescribed condition, for this value in fact gives

$$
H(\alpha U+6 \beta H U)=-x y z,=0,
$$

a system of three lines. We find, moreover,

$$
P(\alpha U+6 \beta H U)=P\left(x^{3}+y^{3}+z^{3}\right),=-\xi \eta \zeta
$$

and

$$
P\{6 H(\alpha U+6 \beta H U)\}=P(-6 x y z),=-4 \xi \eta \zeta,
$$

the latter equation being obtained by first neglecting all but the highest power of $l$ in the expression of $P U$, and then writing $l=-1$ : we have also $T(\alpha U+6 \beta H U)=1$. Substituting the above values, the curve of the sixth class is

$$
\xi \eta \zeta\{-4 T \cdot P U+P(6 H U)\}=0 ;
$$

or throwing out the factor $\xi \eta \zeta$, we have the curve of the third class,

$$
-4 T . P U+P(6 H U)=0 .
$$

Now the general expression in my Third Memoir, viz.

$$
P(\alpha U+6 \beta H U)=\left(\alpha^{3}+12 S \alpha \beta^{2}+4 T \beta^{3}\right) P U+\left(\alpha^{2} \beta-4 S \beta^{3}\right) Q U,
$$

putting $\alpha=0, \beta=1$, gives

$$
P(6 H U)=4 T \cdot P U-4 S \cdot Q U,
$$

or what is the same thing,

$$
-4 T \cdot P U+P(6 H U)=-4 S \cdot Q U
$$

and the curve of the third class is therefore the Quippian $Q U=0$. It may be remarked, that for a cubic $U=0$ the Hessian of which breaks up into three lines, the above investigation shows that we have $P U=-\xi \eta \zeta, P(6 H U)=-4 \xi \eta \zeta$, and $T=1$, and consequently that $-4 T \cdot P U+P(6 H U)$ ought to vanish identically; this in fact happens in virtue of the factor $S$ on the right-hand side, the invariant $S$ of a cubic of the form in question being equal to zero; the appearance of the factor $S$ on the right-hand side is thus accounted for $a$ priori.

Article No. 22.-Theorem relating to a line which meets three given conics in six points in involution.

22. The envelope of a line which meets three given conics, the first or conic polars of any three points with respect to the cubic, in six points in involution, is the Pippian.

It is readily seen that if the theorem is true with respect to the three conics,

$$
\frac{d U}{d x}=0, \quad \frac{d U}{d y}=0, \quad \frac{d U}{d z}=0,
$$


it is true with respect to any three conics whatever of the form

$$
\lambda \frac{d U}{d x}+\mu \frac{d U}{d y}+\nu \frac{d U}{d z}=0,
$$

that is, with respect to any three conics, each of them the first or conic polar of some point $(\lambda, \mu, \nu)$ with respect to the cubic. Considering then these three conics, take $\xi x+\eta y+\zeta z=0$ as the equation of the line, and let $(X, Y, Z)$ be the coordinates of a point of intersection with the first conic, we have

$$
\begin{aligned}
& \xi X+\eta Y+\zeta Z=0 \\
& X^{2}+2 l Y Z=0
\end{aligned}
$$

and combining with these a linear. equation

$$
\alpha X+\beta Y+\gamma Z=0,
$$

in which $(\alpha, \beta, \gamma)$ are arbitrary quantities, we have

$$
X: Y: Z=\gamma \eta-\beta \zeta: \alpha \zeta-\gamma \xi: \beta \xi-\alpha \eta
$$

and hence

$$
(\gamma \eta-\beta \zeta)^{2}+2 l(\alpha \zeta-\gamma \xi)(\beta \xi-\alpha \eta)=0,
$$

an equation in $(\alpha, \beta, \gamma)$ which is in fact the equation in line coordinates of the two points of intersection with the first conic. Developing and forming the analogous equations, we find

$$
\begin{aligned}
& \left(-2 l \eta \zeta, \quad \zeta^{2}, \quad \eta^{2},-\eta \zeta-l \xi^{2}, \quad l \xi \eta, \quad l \xi \zeta \quad \gamma(\alpha, \beta, \gamma)^{2}=0\right. \text {, } \\
& \left(\zeta^{2},-2 l \zeta \xi, \quad \xi^{2}, l \xi \eta,-\zeta \xi-l \eta^{2}, \quad l \eta \zeta \quad \gamma \alpha, \beta, \gamma\right)^{2}=0 \text {, } \\
& \left(\eta^{2}, \quad \xi^{2},-2 l \xi \eta, l \zeta \xi, l \eta \zeta,-\xi \eta-l \zeta^{2} \gamma \alpha, \beta, \gamma\right)^{2}=0 \text {, }
\end{aligned}
$$

which are respectively the equations in line coordinates of the three pairs of intersections.

Now combining these equations with the equation $\gamma=0$, we have the equations of the pairs of lines joining the points of intersection with the point $(x=0, y=0)$, and if the six points are in involution, the six lines must also be in involution, or the condition for the involution of the six points is

$$
\left|\begin{array}{ccc}
-2 l \eta \zeta, & \zeta^{2}, & l \xi \zeta \\
\zeta^{2}, & -2 l \zeta \eta, & l \eta \zeta, \\
\eta^{2}, & \xi^{2}, & -\xi \eta-l \zeta^{2}
\end{array}\right|=0
$$

that is,

$$
4 l^{2} \zeta^{2} \xi \eta\left(-\xi \eta-l \zeta^{2}\right)+l \eta^{3} \zeta^{3}+l \xi^{3} \zeta^{3}+2 l^{2} \xi^{2} \eta^{2} \zeta^{2}+2 l^{2} \xi^{2} \eta^{2} \zeta^{2}+\zeta^{4}\left(-\xi \eta-l \zeta^{2}\right)=0
$$

or, reducing and throwing out the factor $\zeta^{3}$, we find

$$
-l\left(\xi^{3}+\eta^{3}+\zeta^{3}\right)+\left(-1+4 l^{3}\right) \xi \eta \zeta=0,
$$

which shows that the line in question is a tangent of the Pippian. 
It is to be remarked that any three conics whatever may be considered as the first or conic polars of three properly selected points with respect to a properly selected cubic curve. The theorem applies therefore to any three conics whatever, but in this case the cubic curve is not given, and the Pippian therefore stands merely for a curve of the third class, and the theorem is as follows, viz. the envelope of a line which meets any three conics in six points in involution, is a curve of the third class.

Article No. 23.-Completion of the theory in Liouville, and comparison with analogous theorems of HESSE.

In order to convert the foregoing theorem into its reciprocal, we must replace the cubic $U=0$ by a curve of the third class, that is we must consider the coordinates which enter into the equation as line coordinates; and it of course follows that the coordinates which enter into the equation $P U=0$ must be considered as point coordinates, that is we must consider the Pippian as a curve of the third order: we have thus the theorem; The locus of a point such that the tangents drawn from it to three given conics (the first or conic poles of any three lines with respect to a curve of the third class) form a pencil in involution, is the Pippian considered as a curve of the third order. This in fact completes the fundamental theorem in my memoirs in Liouville above referred to, and establishes the analogy with Hesse's results in relation to the Hessian; to show this I set out the two series of theorems as follows :

Hesse, in his memoirs On Curves of the Third Order and Curves of the Third Class, Crelle, tt. xxviII. xxxvi. and xxxviII. [1844, 1848, 1849], has shown as follows :

(a) The locus of a point such that its polars with respect to the three conics $X=0, Y=0, Z=0$ (or more generally its polars with respect to all the conics of the series $\lambda X+\mu Y+\nu Z=0)$ meet in a point, is a curve of the third order $V=0$.

$(\beta)$ Conversely, given a curve of the third order $V=0$, there exists a series of conics such that the polars with respect to all the conics of any point whatever of the curve $V=0$, meet in a point.

$(\gamma)$ The equation of any one of the conics in question is

$$
\lambda \frac{d U}{d x}+\mu \frac{d U}{d y}+\nu \frac{d U}{d z}=0,
$$

that is, the conic is the first or conic polar of a point $(\lambda, \mu, \nu)$ with respect to a certain curve of the third order $U=0$; and this curve is determined by the condition that its Hessian is the given curve $V=0$, that is, we have $V=H U$.

( $\delta$ ) The equation $V=H U$ is solved by assuming $U=a V+b H V$, for we have then $H(a V+b H V)=A V+B H V$, where $A, B$ are given cubic functions of $a, b$, and thence $V=H U=A V+B H V$, or $A=1, B=0$; the latter equation gives what is alone important, the ratio $a: b$; and it thus appears that there are three distinct series of conics, 
each of them having the above-mentioned relation to the given curve of the third order $V=0$.

In the memoirs in Liouville above referred to, I have in effect shown that-

$\left(\alpha^{\prime}\right)$ The locus of a point such that the tangents from it to three conics, represented in line coordinates by the equations $X=0, Y=0, Z=0$ (or more generally with respect to any three conics of the series $\lambda X+\mu Y+\nu Z=0$ ) form a pencil in involution, is a curve of the third order $V=0$.

$\left(\beta^{\prime}\right)$ Conversely, given a curve of the third order $V=0$, there exists a series of conics such that the tangents from any point whatever of the curve to any three of the conics, form a pencil in involution.

Now, considering the coordinates which enter into the equation of the Pippian as point coordinates, and consequently the Pippian as a curve of the third order, I am able to add as follows:

$\left(\gamma^{\prime}\right)$ The equation in line coordinates of any one of the conics in question is

$$
\lambda \frac{d U}{d \xi}+\mu \frac{d U}{d \eta}+\nu \frac{d U}{d \zeta}=0
$$

that is, the conic is the first or conic polar of a line $(\lambda, \mu, \nu)$ with respect to a certain curve of the third class $U=0$; and this curve is determined by the condition that its Pippian is the given curve of the third order $V=0$, that is, we have $V=P U$.

$\left(\delta^{\prime}\right)$ The equation $V=P U$ is solved by assuming $U=a P V+b Q V$, for we have then $P(a P V+b Q V)=A V+B H V$, where $A$ and $B$ are given cubic functions of $a, b$; and thence $V=P U=A V+B H V$, or $A=1, B=0$; the latter equation gives what is alone important, the ratio $a: b$; and it thus appears that there are three distinct curves of the third class $U=0$, and therefore (what indeed is shown in the Memoirs in Liouville) three distinct series of conics having the above-mentioned relation to the given curve of the third order $V=0$.

It is hardly necessary to remark that the preceding theorems, although precisely analogous to those of Hesse, are entirely distinct theorems, that is the two series are not connected together by any relation of reciprocity.

Article Nos. 24 to 28.-Various investigations and theorems.

24. Reverting to the theorem (No. 18), that the lineo-polar envelope of the line $E F$ is the pair of lines $O E, O F$; the line $E F$ is any tangent of the Pippian, hence the theorem includes the following one: 
The lineo-polar envelope with respect to the cubic, of any tangent of the Pippian, is a pair of lines.

And conversely,

The Pippian is the envelope of a line such that the lineo-polar envelope of the line with respect to the cubic is a pair of lines.

It is I think worth while to give an independent proof. It has been shown that the equation of the lineo-polar envelope with respect to the cubic, of the line $\xi x+\eta y+\zeta z=0$ (where $\xi, \eta, \zeta$ are arbitrary quantities), is

$\left(-l^{2} \xi^{2}-2 l \eta \zeta, \quad-l^{2} \eta^{2}-2 l \zeta \xi, \quad-l^{2} \zeta^{2}-2 l \xi \eta, \quad \frac{1}{2} \xi^{2}+l^{2} \eta \zeta, \quad \frac{1}{2} \eta^{2}+l^{2} \zeta \xi, \quad \frac{1}{2} \zeta^{2}+l^{2} \xi \eta \gamma x, y, x\right)^{2}=0 ;$

and representing this equation by

$$
\frac{1}{2}\left(a, b, c, f, g, h \gamma(x, y, z)^{2}=0,\right.
$$

we find

$$
\begin{aligned}
& b c-f^{2}=\xi\left(-\xi^{3}+8 l^{3} \eta^{3}+8 l^{3} \zeta^{3}+12 l^{2} \xi \eta \zeta\right), \\
& c a-g^{2}=\eta\left(8 l^{3} \xi^{3}-\eta^{3}+8 l^{3} \zeta^{3}+12 l^{2} \xi \eta \zeta\right), \\
& a b-h^{2}=\zeta\left(8 l^{3} \xi^{3}+8 l^{3} \eta^{3}-\zeta^{3}+12 l^{2} \xi \eta \zeta\right), \\
& g h-a f=\xi\left(2 l^{2}\left(\xi^{3}+\eta^{3}+\zeta^{3}\right)+4 l\left(1+2 l^{3}\right) \xi \eta \zeta\right)+\left(1+8 l^{3}\right) \eta^{2} \zeta^{2}, \\
& h f-b g=\eta\left(2 l^{2}\left(\xi^{3}+\eta^{3}+\zeta^{3}\right)+4 l\left(1+2 l^{3}\right) \xi \eta \zeta\right)+\left(1+8 l^{3}\right) \zeta^{2} \xi^{2}, \\
& f g-c h=\zeta\left(2 l^{2}\left(\xi^{3}+\eta^{3}+\zeta^{3}\right)+4 l\left(1+2 l^{3}\right) \xi \eta \zeta\right)+\left(1+8 l^{3}\right) \xi^{2} \eta^{2}
\end{aligned}
$$

and after all reductions,

$$
\begin{aligned}
& a b c-a f^{2}-b g^{2}-c h^{2}+2 f g h \\
& =\left[-l\left(\xi^{3}+\eta^{3}+\zeta^{3}\right)+\left(-1+4 l^{3}\right) \xi \eta \zeta\right]^{2}=(P U)^{2},
\end{aligned}
$$

or the condition in order that the conic may break up into a pair of lines is $P U=0$.

25. The following formulæ are given in connexion with the foregoing investigation, but I have not particularly considered their geometrical signification. The lineo-polar envelope of an arbitrary line $\xi x+\eta y+\zeta z=0$, with respect to the cubic

$$
x^{3}+y^{3}+z^{3}+6 l x y z=0,
$$

has been represented by

$$
(a, b, c, f, g, h \chi x, y, z)^{2}=0 ;
$$

and if in like manner we represent the lineo-polar envelope of the same line, with respect to a syzygetic cubic

by

$$
x^{3}+y^{3}+z^{3}+6 l^{\prime} x y z=0,
$$

$$
\left(a^{\prime}, b^{\prime}, c^{\prime}, f^{\prime}, g^{\prime}, h^{\prime} \chi x, y, z\right)^{2}=0,
$$

C. II. 
then we have

$$
\begin{aligned}
a^{\prime}\left(b c-f^{2}\right)+b^{\prime}\left(c a-g^{2}\right)+c^{\prime}\left(a b-h^{2}\right)+2 f^{\prime \prime}(g h-a f)+2 g^{\prime}(h f-b g)+2 h^{\prime}(f g-c h) & \left(l^{\prime 2}+2 l^{2}\right)\left(\xi^{3}+\eta^{3}+\zeta^{3}\right)^{2} \\
= & +\left(2 l^{\prime}+4 l-32 l^{3} l^{\prime}+8 l^{4}\right)\left(\xi^{3}+\eta^{3}+\zeta^{3}\right) \xi \eta \zeta \\
& +\left(24 l l^{\prime 2}+48 l^{4} l^{\prime 2}-72 l^{2} l^{\prime}+24 l^{3}+3\right) \xi^{2} \eta^{2} \zeta^{2},
\end{aligned}
$$

which may be verified by writing $l^{\prime}=l$, in which case the right-hand side becomes as it should do, $3(P U)^{2}$. If $l^{\prime}=-\frac{1+2 l^{3}}{6 l^{2}}$, that is, if the syzygetic cubic be the Hessian, then the formula becomes

$$
a^{\prime}\left(b c-f^{2}\right)+\& c .=\frac{1}{36 l^{4}}\left\{\begin{array}{c}
\left(1+4 l^{3}+76 l^{6}\right)\left(\xi^{3}+\eta^{3}+\zeta^{3}\right)^{2} \\
+12 l^{2}\left(-1+26 l^{3}+56 l^{6}\right)\left(\xi^{3}+\eta^{3}+\zeta^{3}\right) \xi \eta \zeta \\
+12 l\left(2+57 l^{3}+168 l^{6}+16 l^{10}\right) \xi^{2} \eta^{2} \zeta^{2}
\end{array}\right\}
$$

which is equal to

$$
\frac{1}{36 l^{4}}\left\{\overline{Q U}^{2}-24 S \cdot \overline{P U}^{2}\right\}
$$

26. The equation

$$
\left(b c^{\prime}+b^{\prime} c-2 f f^{\prime}, \ldots g h^{\prime}+g^{\prime} h-a f^{\prime}-a^{\prime} f, \ldots \gamma \xi, \eta, \zeta\right)^{2}=0
$$

is the equation in line coordinates of a conic, the envelope of the line which cuts harmonically the conics

$$
\begin{aligned}
& \left(a, b, c, f, g, h \gamma(x, y, z)^{2}=0,\right. \\
& \left(a^{\prime}, b^{\prime}, c^{\prime}, f^{\prime}, g^{\prime}, h^{\prime} \chi x, \bar{y}, z\right)^{2}=0 ;
\end{aligned}
$$

and if $a, b, \& c ., a^{\prime}$, \&c. have the values before given to them, then the coefficients of the equation are

$$
\begin{aligned}
& b c^{\prime}+b^{\prime} c-2 f f^{\prime}=\xi\left\{-\xi^{3}+4 l l^{\prime}\left(l+l^{\prime}\right)\left(\eta^{3}+\zeta^{3}\right)+\left(16 l l^{\prime}-2 l^{2}-2 l^{\prime 2}\right) \xi \eta \zeta,\right. \\
& c a^{\prime}+c^{\prime} a-2 g g^{\prime}=\eta\left\{-\eta^{3}+4 l l^{\prime}\left(l+l^{\prime}\right)\left(\zeta^{3}+\xi^{3}\right)+\left(16 l l^{\prime}-2 l^{2}-2 l^{\prime 2}\right) \xi \eta \zeta,\right. \\
& a b^{\prime}+a^{\prime} b-2 h h^{\prime}=\zeta\left\{-\zeta^{3}+4 l l^{\prime}\left(l+l^{\prime}\right)\left(\xi^{3}+\eta^{3}\right)+\left(16 l l^{\prime}-2 l^{2}-2 l^{\prime 2}\right) \xi \eta \zeta,\right. \\
& g h^{\prime}+g^{\prime} h-a f^{\prime}-a^{\prime} f=\xi\left\{\left(l^{2}+l^{\prime 2}\right)\left(\xi^{3}+\eta^{3}+\zeta^{3}\right)+\left(2 l+2 l^{\prime}+8 l^{2} l^{\prime 2}\right) \xi \eta \zeta\right\}+\left(1+4 l l^{\prime}\left(l+l^{\prime}\right)\right) \eta^{2} \zeta^{2}, \\
& h f^{\prime}+h^{\prime} f-b g^{\prime}-b^{\prime} g=\eta\left\{\left(l^{2}+l^{\prime 2}\right)\left(\xi^{3}+\eta^{3}+\zeta^{3}\right)+\left(2 l+2 l^{\prime}+8 l^{2} l^{\prime 2}\right) \xi \eta \zeta\right\}+\left(1+4 l l^{\prime}\left(l+l^{\prime}\right)\right) \zeta^{2} \xi^{2}, \\
& f g^{\prime}+f^{\prime} g-c h^{\prime}-c^{\prime} h=\zeta\left\{\left(l^{2}+l^{\prime 2}\right)\left(\xi^{3}+\eta^{3}+\zeta^{3}\right)+\left(2 l+2 l^{\prime}+8 l^{2} l^{\prime 2}\right) \xi \eta \zeta\right\}+\left(1+4 l l^{\prime}\left(l+l^{\prime}\right)\right) \xi^{2} \eta^{2}:
\end{aligned}
$$

and we thence obtain

$$
\begin{aligned}
\left(b c^{\prime}+b^{\prime} c-2 f f^{\prime}, \ldots, g h^{\prime}+g^{\prime} h-a f^{\prime}-a^{\prime} f, . . \gamma \xi, \eta, \zeta\right)^{2}= & \\
& -\left(\xi^{3}+\eta^{3}+\zeta^{3}\right)^{2} \\
& +\left(l^{2}+l^{\prime 2}+16 l l^{\prime}\right)\left(\xi^{3}+\eta^{3}+\zeta^{3}\right) \xi \eta \zeta \\
& +\left(6 l+6 l^{\prime}+24 l^{2} l^{\prime 2}\right) \xi^{2} \eta^{2} \zeta^{2} \\
& +\left(4+16\left(l^{2} l^{\prime}+l l^{\prime 2}\right)\right)\left(\eta^{3} \zeta^{3}+\zeta^{3} \xi^{3}+\xi^{3} \eta^{3}\right),=0
\end{aligned}
$$


as the condition which expresses that a line $\xi x+\eta y+\zeta z=0$ cuts harmonically its lineo-polar envelopes with respect to the cubic and with respect to a syzygetic cubic.

27. To find the locus of a point such that its second or line polar with respect to the cubic may be a tangent of the Pippian. Let the coordinates of the point be $(x, y, z)$; then if $\xi x+\eta y+\zeta z=0$ be the equation of the polar, we have

$$
\xi: \eta: \zeta=x^{2}+2 l y z: y^{2}+2 l z x: z^{2}+2 l x y,
$$

and the line in question being a tangent to the Pippian,

$$
-l\left(\xi^{3}+\eta^{3}+\zeta^{3}\right)+\left(-1+4 l^{3}\right) \xi \eta \zeta=0 .
$$

But the preceding values give

$$
\begin{array}{rrrr}
\xi^{3}+\eta^{3}+\zeta^{3} & = & \left(x^{3}+y^{3}+z^{3}\right)^{2}+6 l\left(x^{3}+y^{3}+z^{3}\right) x y z+ & 36 l^{2} x^{2} y^{2} z^{2}+\left(-2+8 l^{3}\right)\left(y^{3} z^{3}+z^{3} x^{3}+x^{2} y^{3}\right) \\
\xi \eta \zeta & = & 4 l^{2}\left(x^{3}+y^{3}+z^{3}\right) x y z+\left(1+8 l^{3}\right) x^{2} y^{2} z^{2}+ & 2 l\left(y^{3} z^{3}+z^{3} x^{3}+x^{3} y^{3}\right)
\end{array}
$$

and we have therefore

$$
l\left(x^{3}+y^{3}+z^{3}\right)^{2}+\left(10 l^{2}-16 l^{5}\right)\left(x^{3}+y^{3}+z^{3}\right) x y z+\left(1+40 l^{3}-32 l^{6}\right) x^{2} y^{2} z^{2}=0 ;
$$

or introducing $U, H U$ in place of $x^{3}+y^{3}+z^{3}$, xyz, the equation becomes

$$
-S \cdot U^{2}+(H U)^{2}=0
$$

which is the equation of the locus in question.

28. The locus of a point such that its second or line polar with respect to the cubic is a tangent of the Quippian, is found in like manner by substituting the lastmentioned values of $\xi, \eta, \zeta$ in the equation

$$
Q U=\left(1-10 l^{3}\right)\left(\xi^{3}+\eta^{3}+\zeta^{3}\right)-6 l^{2}\left(5+4 l^{3}\right) \xi \eta \zeta .
$$

We find as the equation of the locus,

$$
\begin{aligned}
\left(1-10 l^{3}\right)\left(x^{3}+y^{3}+z^{3}\right)^{2}+6 l\left(1-30 l^{3}-16 l^{6}\right)\left(x^{3}+y^{3}+z^{3}\right) x y z & +6 l^{2}\left(1-104 l^{3}-32 l^{6}\right) x^{2} y^{2} z^{2} \\
- & 2\left(1+8 l^{3}\right)^{2}\left(y^{3} z^{3}+z^{3} x^{3}+x^{3} y^{3}\right)=0
\end{aligned}
$$

where the function on the left-hand side is the octicovariant $\Theta_{11} U$ of my Third Memoir, the covariant having been in fact defined so as to satisfy the condition in question. And I have given in the memoir the following expression for $\Theta_{1 /} U$, viz.

$$
\begin{aligned}
& \Theta_{\text {"I }} U=\left(1-16 l^{3}-6 l^{6}\right) U^{2} \\
& +(6 l \quad) U \cdot H U \\
& +\left(6 l^{2} \quad\right)(H U)^{2} \\
& -\quad 2\left(1+8 l^{3}\right)^{2}\left(y^{3} z^{3}+z^{3} x^{3}+x^{3} y^{3}\right) \text {. }
\end{aligned}
$$


Article Nos. 29 to 31. -Formulce for the intersection of a cubic curve and a line.

29. If the line $\xi x+\eta y+\zeta z=0$ meet the cubic

$$
x^{3}+y^{3} z^{3}+6 l x y z=0
$$

in the points

$$
\left(x_{1}, y_{1}, z_{1}\right), \quad\left(x_{2}, y_{2}, z_{2}\right), \quad\left(x_{3}, y_{3}, z_{3}\right),
$$

then we have

$$
x_{1} x_{2} x_{3}: y_{1} y_{2} y_{3}: z_{1} z_{2} z_{3}=\eta^{3}-\zeta^{3}: \zeta^{3}-\xi^{3}: \xi^{3}-\eta^{3} .
$$

It will be convenient to represent the equation of the cubic by the abbreviated notation $\left(1,1,1, l \gamma(x, y, z)^{3}=0\right.$; we have the two equations

$$
\begin{aligned}
& \left(1,1,1, l \gamma(x, y, z)^{3}=0,\right. \\
& \xi x+\eta y+\zeta z \quad=0
\end{aligned}
$$

and if to these we join a linear equation with arbitrary coefficients,

$$
\alpha x+\beta y+\gamma z=0,
$$

then the second and third equations give

$$
x: y: z=\beta \zeta-\gamma \eta: \gamma \xi-\alpha \zeta: \alpha \eta-\beta \xi ;
$$

and substituting these values in the first equation, we obtain the resultant of the system. But this resultant will also be obtained by substituting, in the third equation, a system of simultaneous roots of the first and second equations, and equating to zero the product of the functions so obtained ${ }^{1}$. We must have therefore

$$
(1,1,1, l \gamma \beta \zeta-\gamma \eta, \gamma \xi-\alpha \zeta, \alpha \eta-\beta \xi)^{3}=\left(\alpha x_{1}+\beta y_{1}+\gamma z_{1}\right)\left(\alpha x_{2}+\beta y_{2}+\gamma z_{2}\right)\left(\alpha x_{3}+\beta y_{3}+\gamma z_{3}\right) \text {; }
$$

and equating the coefficients of $\alpha^{3}, \beta^{3}, \gamma^{3}$, we obtain the above-mentioned relations.

30. If a tangent to the cubic

$$
x^{3}+y^{3}+z^{3}+6 l x y z=0
$$

at a point $\left(x_{1}, y_{1}, z_{1}\right)$ of the cubic meet the cubic in the point $\left(x_{3}, y_{3}, z_{3}\right)$, then

$$
x_{3}: y_{3}: z_{3}=x_{1}\left(y_{1}^{3}-z_{1}^{3}\right): y_{1}\left(z_{1}^{3}-x_{1}^{3}\right): z_{1}\left(\dot{x}_{1}{ }^{3}-y_{1}{ }^{3}\right) \text {. }
$$

For if the equation of the tangent is $\xi x+\eta y+\zeta z=0$, then

$$
x_{1}^{2} x_{3}: y_{1}^{2} y_{3}: z_{1}^{2} z_{3}=\eta^{3}-\zeta^{3}: \zeta^{3}-\xi^{3}: \xi^{3}-\eta^{3},
$$

and

$$
\xi: \eta: \zeta=x_{1}^{2}+2 l y_{1} z_{1}: y_{1}^{2}+2 l z_{1} x_{1}: z_{1}{ }^{2}+2 l x_{1} y_{1} .
$$

1 This is in fact the general process of elimination given in Schläfli's Memoir, "Ueber die Resultante einer Systemes mehrerer algebraischer Gleichungen,". Vienna Trans. 1852. [But the process was employed much earlier, by Poisson.] 
These values give

$$
\begin{aligned}
\eta^{3}-\zeta^{3} & =\left(y_{1}^{3}-z_{1}^{3}\right)\left(y_{1}^{3}+z_{1}^{3}+6 l x_{1} y_{1} z_{1}-8 l^{3} x_{1}^{3}\right) \\
& =\left(y_{1}{ }^{3}-z_{1}^{3}\right) \times-\left(1+8 l^{3}\right) x_{1}^{3},
\end{aligned}
$$

since $\left(x_{1}, y_{1}, z_{1}\right)$ is a point of the cubic; and forming in like manner the values of $\xi^{3}-\xi^{3}$ and $\xi^{3}-\eta^{3}$, we obtain the theorem.

31. The preceding values of $\left(x_{3}, y_{3}, z_{3}\right)$ ought to satisfy

$$
\begin{aligned}
& \left(x_{1}{ }^{2}+2 l y_{1} z_{1}\right) x_{3}+\left(y_{1}{ }^{2}+2 l z_{1} x_{1}\right) y_{3}+\left(z_{1}{ }^{2}+2 l x_{1} y_{1}\right) z_{3}=0, \\
& x_{3}{ }^{2}+y_{3}{ }^{2}+z_{3}{ }^{2}+6 l x_{3} y_{3} z_{3}=0
\end{aligned}
$$

in fact the first equation is satisfied identically, and for the second equation we obtain

$$
\begin{aligned}
x_{3}{ }^{2}+y_{3}{ }^{2}+z_{3}{ }^{2} & =x_{1}{ }^{3}\left(y_{1}{ }^{3}-z_{1}{ }^{3}\right)^{3}+y_{1}{ }^{3}\left(z_{1}{ }^{3}-x_{1}\right)^{3}+z_{1}{ }^{3}\left(x_{1}{ }^{3}-y_{1}{ }^{3}\right)^{2} \\
& =-x_{1}{ }^{9}\left(y_{1}{ }^{3}-z_{1}{ }^{3}\right)-y_{1}{ }^{9}\left(z_{1}{ }^{3}-x_{1}{ }^{3}\right)-z_{1}{ }^{9}\left(x_{1}{ }^{3}-y_{1}{ }^{3}\right) \\
& =\left(x_{1}{ }^{3}+y_{1}{ }^{3}+z_{1}^{3}\right)\left(y_{1}{ }^{3}-z_{1}{ }^{3}\right)\left(z_{1}{ }^{3}-x_{1}{ }^{3}\right)\left(x_{1}{ }^{3}-y_{1}{ }^{3}\right), \\
x_{3} y_{3} z_{3} & =x_{1} y_{1} z_{1}\left(y_{1}{ }^{3}-z_{1}{ }^{3}\right)\left(z_{1}{ }^{3}-x_{1}{ }^{3}\right)\left(x_{1}{ }^{3}-y_{1}{ }^{3}\right),
\end{aligned}
$$

and consequently

$$
x_{3}{ }^{3}+y_{3}{ }^{3}+z_{3}{ }^{3}+6 l x_{3} y_{3} z_{3}=\left(x_{1}{ }^{3}+y_{1}{ }^{3}+z_{1}{ }^{3}+6 l x_{1} y_{1} z_{1}\right)\left(y_{1}{ }^{3}-z_{1}{ }^{3}\right)\left(z_{1}{ }^{3}-x_{1}^{3}\right)\left(x_{1}{ }^{3}-y_{1}{ }^{3}\right)=0,
$$

which verifies the theorem. It is proper to add (the remark was made to me by Professor Sylvester) that the foregoing values

$$
x_{3}: y_{3}: z_{3}=x_{1}\left(y_{1}^{3}-z_{1}^{3}\right): y_{1}\left(z_{1}^{3}-x_{1}^{3}\right): z_{1}\left(x_{1}^{3}-y_{1}^{3}\right)
$$

satisfy identically the relation

$$
\frac{x_{3}{ }^{3}+y_{3}{ }^{3}+z_{3}{ }^{3}}{x_{3} y_{3} z_{3}}=\frac{x_{1}{ }^{3}+y_{1}{ }^{3}+z_{1}{ }^{2}}{x_{1} y_{1} z_{1}} .
$$

Article Nos. 32 to 34.-Formulce for the Satellite line and point.

32. The line $\xi x+\eta y+\zeta z=0$ meets the cubic

$$
x^{3}+y^{3}+z^{3}+6 l x y z=0
$$

in three points, and the tangents to the cubic at these points meet the cubic in three points lying in a line, which has been called the Satellite line of the given line.

To find the equation of the satellite line; suppose that $\left(x_{1}, y_{1}, z_{1}\right),\left(x_{2}, y_{2}, z_{2}\right)$, $\left(x_{3}, y_{3}, z_{3}\right)$ are the coordinates of the point in which the given line meets the cubic; then we have, as before,

$(1,1,1, l \gamma \beta \zeta-\gamma \eta, \gamma \xi-\alpha \zeta, \alpha \eta-\beta \xi)^{3}=\left(\alpha x_{1}+\beta y_{1}+\gamma z_{1}\right)\left(\alpha x_{2}+\beta y_{2}+\gamma z_{2}\right)\left(\alpha x_{3}+\beta y_{3}+\gamma z_{3}\right)$. 
The equation of the three tangents is

$$
\left.\begin{array}{rl}
\Pi & =\left[\left(x_{1}^{2}+2 l y_{1} z_{1}\right) x+\left(y_{1}{ }^{2}+2 l z_{1} x_{1}\right) y+\left(z_{1}{ }^{2}+2 l x_{1} y_{1}\right) z\right] \\
& \times\left[\left(x_{2}{ }^{2}+2 l y_{2} z_{2}\right) x+\left(y_{2}{ }^{2}+2 l z_{2} x_{2}\right) y+\left(z_{2}{ }^{2}+2 l x_{2} y_{2}\right) z\right] \\
& \times\left[\left(x_{3}{ }^{2}+2 l y_{3} z_{3}\right) x+\left(y_{3}{ }^{2}+2 l z_{3} x_{3}\right) y+\left(z_{3}{ }^{2}+2 l x_{3} y_{3}\right) z\right]
\end{array}\right\}=0,
$$

and if we put

$$
F=\left(\xi^{3}+\eta^{3}+\zeta^{3}\right)^{2}-24 l^{2}\left(\xi^{3}+\eta^{3}+\zeta^{3}\right) \xi \eta \zeta+\left(-24 l-48 l^{4}\right) \xi^{2} \eta^{2} \zeta^{2}+\left(-4+32 l^{3}\right)\left(\eta^{3} \zeta^{3}+\zeta^{3} \xi^{3}+\xi^{3} \eta^{3}\right),
$$

( $F$ is the reciprocant $F U$ of my Third Memoir), then we have identically

$$
F \cdot U-\Pi=(\xi x+\eta y+\zeta z)^{2}\left(\xi^{\prime} x+\eta^{\prime} y+\zeta^{\prime} z\right),
$$

and the equation of the satellite line is $\xi^{\prime} x+\eta^{\prime} y+\zeta^{\prime} z=0$. In fact the geometrical theory shows that we must have

$$
F \cdot U-N \Pi=(\xi x+\eta y+\zeta z)^{2}\left(\xi^{\prime} x+\eta^{\prime} y+\zeta^{\prime} z\right)
$$

and it is then clear that $N$ is a mere number. To determine its value in the most simple manner, write $l=0, y=0, x=\zeta, z=-\xi$, we have then $F \cdot U-N \Pi=0$, where

$$
F=\xi^{6}+\eta^{6}+\zeta^{6}-2 \eta^{3} \zeta^{3}-2 \zeta^{3} \xi^{3}-2 \xi^{3} \eta^{3}, \quad U=\zeta^{3}-\xi^{3} .
$$

The value of $\Pi$ is $\Pi=F$. $U$, and we thus obtain $N=1$. For, substituting the above values,

$$
\begin{aligned}
\Pi= & \left(x_{1}^{2} \zeta-z_{1}^{2} \xi\right)\left(x_{2}^{2} \zeta-z_{2}^{2} \xi\right)\left(x_{3}^{2} \zeta-z_{3}^{2} \xi\right) \\
= & \zeta^{3} x_{1}^{2} x_{2}^{2} x_{3}^{2} \\
& -\zeta^{2} \xi\left(x_{1}^{2} x_{2}^{2} x_{3}^{2}+\& c .\right) \\
& +\zeta^{2}\left(x_{1} z_{2}^{2} z_{3}^{2}+\& c .\right) \\
& -\xi^{3} z_{1}^{2} z_{2}^{2} z_{3}^{2},
\end{aligned}
$$

and we have

$$
\begin{aligned}
& x_{1} x_{2} x_{3}=\eta^{3}-\zeta^{3}, \\
& x_{1} x_{2} z_{3}+\& c .=3 \zeta^{2} \xi \\
& x_{1} z_{2} z_{3}+\& c . \\
& z_{1} z_{2} z_{3}=-3 \xi^{2}, \\
& =\xi^{3}-\eta^{3},
\end{aligned}
$$

and thence

$$
\begin{aligned}
& x_{1}^{2} x_{2}^{2} z_{3}^{2}+\& c .=9 \zeta^{4} \xi^{2}+6 \zeta^{2}\left(\eta^{3}-\zeta^{3}\right)=3 \zeta^{4} \xi^{2}+6 \zeta^{2} \eta^{3} \\
& x_{1}^{2}{z_{2}}_{2}^{2} z_{3}^{2}+\& c .=9 \zeta^{2} \xi^{4}-6 \zeta^{2} \xi\left(\xi^{3}-\eta^{3}\right)=3 \zeta^{2} \xi^{4}+6 \zeta^{2} \xi \eta^{2}
\end{aligned}
$$

and consequently

$$
\begin{aligned}
\Pi= & \zeta^{3}\left(\eta^{3}-\zeta^{3}\right)^{2} \\
& -\zeta^{2} \xi \cdot 3 \zeta \xi^{2}\left(\zeta^{3}+\eta^{3}\right) \\
& +\zeta^{2} \cdot 3 \zeta^{2} \xi\left(\xi^{3}+\eta^{3}\right) \\
& -\xi^{3}\left(\xi^{3}-\eta^{3}\right)^{2} \\
= & \left(\zeta^{3}-\xi^{3}\right)\left(\xi^{6}+\eta^{6}+\zeta^{6}-2 \zeta^{3} \eta^{3}-2 \zeta^{3} \xi^{3}-2 \xi^{3} \eta^{3}\right) .
\end{aligned}
$$


Now considering the equation

$$
F \cdot U-\Pi=(\xi x+\eta y+\zeta z)^{2}\left(\xi^{\prime} x+\eta^{\prime} y+\zeta^{\prime} z\right),
$$

in order to find $\xi^{\prime}, \eta^{\prime}, \zeta^{\prime}$ it will be sufficient to find the coefficients of $x^{3}, y^{3}, z^{3}$ in the function on the left-hand side of the equation. The coefficient of $x^{3}$ in $\Pi$ is

$$
\begin{aligned}
& \left(x_{1}^{2}+2 l y_{1} z_{1}\right)\left(x_{2}^{2}+2 l z_{2} x_{2}\right)\left(x_{3}^{2}+2 l x_{3} y_{3}\right) \\
& =\quad x_{1}^{2} x_{2}^{2} x_{3}^{2} \\
& \quad+2 l\left(x_{1}^{2} x_{2}^{2} y_{3} z_{3}+\& \mathrm{c} .\right) \\
& +4 l^{2}\left(x_{1}^{2} y_{2} z_{2} y_{3} z_{3}+\& \mathrm{c} .\right) \\
& +8 l^{3} \quad y_{1} y_{2} y_{3} z_{1} z_{2} z_{3}
\end{aligned}
$$

and it is easy to see that representing the function

$$
(1,1,1, l \gamma \beta \zeta-\gamma \eta, \gamma \xi-\alpha \zeta, \alpha \eta-\beta \xi)^{3}
$$

by

$$
\left(\mathrm{a}, \mathrm{b}, \mathrm{c}, \mathrm{f}, \mathrm{g}, \mathrm{h}, \mathrm{i}, \mathrm{j}, \mathrm{k}, \mathrm{l} \gamma(\alpha, \beta, \gamma)^{3}\right. \text {, }
$$

the symmetrical functions can be expressed in terms of the quantities a, b, \&c., and that the preceding value of the coefficient of $x^{3}$ in $\Pi$ is

$$
\begin{aligned}
& a^{2} \\
+ & 2 l(9 h j-6 a l) \\
+ & 4 l^{2}\left(6 g k-3 f j-3 h i+3 l^{2}\right) \\
+ & 8 l^{3} \text { bc }
\end{aligned}
$$

and substituting for a, \&c. their values, this becomes

$$
\begin{aligned}
& \left(\eta^{3}-\zeta^{3}\right)^{2} \\
+2 l & \left\{-9\left(\xi \eta^{2}+2 l \eta \zeta^{2}\right)\left(\zeta^{2} \xi+2 l \zeta \eta^{2}\right)\right\} \\
+ & 4 l^{2}\left\{-6\left(\zeta \xi^{2}+2 l \xi \eta^{2}\right)\left(\xi^{2} \eta+2 l \xi \zeta^{2}\right)\right. \\
& +3\left(\eta \zeta^{2}+2 l \zeta \xi^{2}\right)\left(\zeta^{2} \xi+2 l \zeta \eta^{2}\right) \\
& \left.+3\left(\xi \eta^{2}+2 l \eta \zeta^{2}\right)\left(\eta^{2} \zeta+2 l \eta \xi^{2}\right)\right\} \\
+ & 8 l^{3}\left(\xi^{3}-\eta^{3}\right)\left(\zeta^{3}-\xi^{3}\right),
\end{aligned}
$$

and reducing, we obtain for the coefficient of $x^{3}$ in $\Pi$ the following expression,

$$
\begin{gathered}
\left(\eta^{3}-\zeta^{3}\right)^{2} \\
-18 l \xi^{2} \eta^{2} \zeta^{2} \\
-24 l^{2}\left(\xi^{3}+\eta^{3}+\zeta^{3}\right) \xi \eta \zeta \\
-24 l^{3}\left(\eta^{3} \zeta^{3}+\zeta^{3} \xi^{3}+\xi^{3} \eta^{3}\right) \\
+8 l^{3}\left(\xi^{3}-\eta^{3}\right)\left(\zeta^{3}-\xi^{3}\right) .
\end{gathered}
$$


Now the coefficient of $x^{3}$ in $F . U$ is simply $F$, which is equal to

$$
\begin{aligned}
& \xi^{6}+\eta^{6}+\zeta^{6}-2 \eta^{3} \zeta^{3}-2 \zeta^{3} \xi^{3}-2 \xi^{3} \eta^{3} \\
- & 24 l \quad \xi^{2} \eta^{2} \zeta^{2} \\
- & 24 l^{2}\left(\xi^{3}+\eta^{3}+\zeta^{3}\right) \xi \eta \zeta \\
- & 32 l^{3}\left(\eta^{3} \zeta^{3}+\zeta^{3} \xi^{3}+\xi^{3} \eta^{3}\right) \\
- & 48 l^{4} \xi^{2} \eta^{2} \zeta^{2}
\end{aligned}
$$

and subtracting, the coefficient of $x^{3}$ in $F . U-\Pi$ is

$$
\begin{aligned}
& \xi^{6}-2 \xi^{3} \eta^{3}-2 \xi^{3} \zeta^{3} \\
-6 l \quad \xi^{2} \eta^{2} \zeta^{2} & \\
- & 8 l^{3}\left(\eta^{3} \zeta^{3}+\zeta^{3} \xi^{3}+\xi^{3} \eta^{3}\right) \\
- & 8 l^{3}\left(\xi^{3}-\eta^{3}\right)\left(\zeta^{3}-\xi^{3}\right) \\
- & 48 l^{4} \xi^{2} \eta^{2} \zeta^{2},
\end{aligned}
$$

which is equal to

$$
\left(1+8 l^{3}\right) \xi^{2}\left(\xi^{4}-2 \xi \eta^{3}-2 \xi \zeta^{3}-6 l \eta^{2} \zeta^{2}\right) .
$$

The expression last written down is therefore the value of $\xi^{2} \xi^{\prime}$, or dividing by $\xi^{2}$ we have $\xi^{\prime}$, and then the values of $\eta^{\prime}, \zeta^{\prime}$ are of course known, and we obtain the identical equation

$$
\begin{aligned}
& F \cdot U-\Pi= \\
& \left(1+8 l^{3}\right)(\xi+\eta y+\zeta z)^{2}\left\{\begin{array}{r}
\left(\xi^{4}-2 \xi \eta^{3}-2 \xi \xi^{3}-6 l \eta^{2} \zeta^{2}\right) x \\
+\left(\eta^{4}-2 \eta \zeta^{3}-2 \eta \xi^{3}-6 l \zeta^{2} \xi^{2}\right) y \\
+\left(\zeta^{4}-2 \zeta \xi^{3}-2 \zeta \eta^{3}-6 l \xi^{2} \eta^{2}\right) z
\end{array}\right\}
\end{aligned}
$$

and the second factor equated to zero is the equation of the satellite line of $\xi x+\eta y+\zeta z=0$.

33. The point of intersection of the line $\xi x+\eta y+\zeta z=0$ with the satellite line $\xi^{\prime} x+\eta^{\prime} y+\zeta^{\prime} z=0$ is the satellite point of the former line; and the coordinates of the satellite point are at once found to be

$$
\begin{aligned}
x: y: z & =\left(\eta^{3}-\zeta^{3}\right)\left(\eta \zeta+2 l \xi^{2}\right) \\
& :\left(\zeta^{3}-\xi^{3}\right)\left(\zeta \xi+2 l \eta^{2}\right) \\
& :\left(\xi^{3}-\eta^{3}\right)\left(\xi \eta+2 l \zeta^{2}\right) .
\end{aligned}
$$

34. If the primary line $\xi x+\eta y+\zeta z=0$ is a tangent to the cubic, then $\left(x_{1}, y_{1}, z_{1}\right)$ being the coordinates of the point of contact, we have

$$
\xi: \eta: \zeta=x_{1}{ }^{2}+2 l y_{1} z_{1}: y_{1}{ }^{2}+2 l z_{1} x_{1}: z_{1}{ }^{2}+2 l x_{1} y_{1} ;
$$


these values give as before

$$
\eta^{3}-\zeta^{3}=-\left(1+8 l^{3}\right) x_{1}^{3}\left(y_{1}^{3}-z_{1}^{3}\right)
$$

and they give also

$$
\eta \zeta+2 l \xi^{2}=\left(1+8 l^{3}\right) y_{1}^{2} z_{1}^{2},
$$

and consequently we obtain

$$
x: y: z=x_{1}\left(y_{1}^{3}-z_{1}^{3}\right): y_{1}\left(z_{1}^{3}-x_{1}^{3}\right): z_{1}\left(x_{1}^{3}-y_{1}^{3}\right),
$$

that is, the satellite point of a tangent of the cubic is the point in which this tangent again meets the cubic.

Article Nos. 35 and 36.-Theorems relating to the satellite point.

35. If the line $\xi x+\eta y+\zeta z=0$ be a tangent of the Pippian, then the locus of the satellite point is the Hessian.

Take $(x, y, z)$ as the coordinates of the satellite point, then we have

$$
\begin{aligned}
x: y: z & =\left(\eta^{3}-\zeta^{3}\right)\left(\eta \zeta+2 l \xi^{2}\right) \\
& :\left(\zeta^{3}-\xi^{3}\right)\left(\zeta \xi+2 l \eta^{2}\right) \\
& :\left(\xi^{3}-\eta^{3}\right)\left(\xi \eta+2 l \zeta^{2}\right)
\end{aligned}
$$

where the parameters $\xi, \eta, \zeta$ are connected by the equation

$$
-l\left(\xi^{3}+\eta^{3}+\zeta^{3}\right)+\left(-1+4 l^{3}\right) \xi \eta \zeta=0 .
$$

We have

$$
\begin{aligned}
y^{3}+z^{3} & =\left(\zeta^{3}-\xi^{3}\right)^{3}\left(\zeta \xi+2 l \eta^{2}\right)^{3} \\
& +\left(\xi^{3}-\eta^{3}\right)^{3}\left(\xi \eta+2 l \zeta^{2}\right)^{3},
\end{aligned}
$$

and it is easy to see that the function on the right-hand side must divide by $\eta^{3}-\zeta^{3}$ : hence $x^{3}+y^{3}+z^{3}$ will also divide by $\eta^{3}-\zeta^{3}$, and consequently by $\left(\eta^{3}-\zeta^{3}\right)\left(\zeta^{3}-\xi^{3}\right)\left(\xi^{3}-\eta^{3}\right)$. We have

$$
\begin{aligned}
\left(y^{3}+z^{3}\right) \div\left(\eta^{3}-\zeta^{3}\right)= & \xi^{3}\left\{\begin{array}{c}
-\zeta^{9}-\zeta^{6} \eta^{3}-\zeta^{3} \eta^{6}-\eta^{9} \\
+3 \xi^{3}\left(\zeta^{6}+\zeta^{3} \eta^{3}+\eta^{6}\right) \\
-3 \xi^{6}\left(\zeta^{3}+\eta^{3}\right) \\
+\xi^{9}
\end{array}\right\} \\
& +6 l \xi^{2} \eta^{2} \zeta^{2}\left\{-\zeta^{6}-\zeta^{3} \eta^{3}-\eta^{6}+3_{2}^{\xi^{3}}\left(\zeta^{3}+\eta^{3}\right)-3 \xi^{6}\right\} \\
& +12 l^{2} \xi \eta \zeta\left\{-\eta^{3} \zeta^{3}\left(\eta^{3}+\zeta^{3}\right)+3 \xi^{3} \eta^{3} \zeta^{3}-\xi^{9}\right\} \\
& +8 l^{3} \quad\left\{-\eta^{6} \zeta^{6}+3 \eta^{3} \zeta^{3} \xi^{6}-\left(\eta^{3}+\zeta^{3}\right) \xi^{9}\right\}
\end{aligned}
$$

and

$$
x^{3} \div\left(\eta^{3}-\zeta^{3}\right)=\left(\eta^{6}-2 \eta^{3} \zeta^{3}+\zeta^{6}\right)\left(\eta^{3} \zeta^{3}+6 l \xi^{2} \eta^{2} \zeta^{2}+12 l^{4} \xi^{4} \eta \zeta+8 l^{3} \xi^{6}\right)
$$

C. II. 
Adding these values and completing the reduction, we find

$$
\begin{aligned}
\left(x^{3}+y^{3}+z^{3}\right) \div\left(\eta^{3}-\zeta^{3}\right)\left(\zeta^{3}-\xi^{3}\right)\left(\xi^{3}-\eta^{3}\right)= & -\xi^{6}-\eta^{6}-\zeta^{6}+2 \eta^{3} \zeta^{3}+2 \zeta^{3} \xi^{3}+2 \xi^{3} \eta^{3} \\
& +18 l \xi^{2} \eta^{2} \zeta^{2} \\
& +12 l^{2}\left(\xi^{3}+\eta^{3}+\zeta^{3}\right) \xi \eta \zeta \\
& +8 l^{3}\left(\eta^{3} \zeta^{3}+\zeta^{3} \xi^{3}+\xi^{3} \eta^{3}\right)
\end{aligned}
$$

and we have also

$$
\begin{aligned}
x y z \div\left(\eta^{3}-\zeta^{3}\right)\left(\zeta^{3}-\xi^{3}\right)\left(\xi^{3}-\eta^{3}\right) \quad & \xi^{2} \eta^{2} \zeta^{2} \\
& +2 l\left(\xi^{3}+\eta^{3}+\zeta^{3}\right) \xi \eta \zeta \\
& +4 l^{2}\left(\eta^{3} \zeta^{3}+\zeta^{3} \xi^{3}+\xi^{3} \eta^{3}\right) \\
& +8 l^{3} \xi^{2} \eta^{2} \zeta^{2}
\end{aligned}
$$

and thence

$$
\begin{aligned}
& \left\{A\left(x^{3}+y^{3}+z^{3}\right)+B x y z\right\} \div\left(\eta^{3}-\zeta^{3}\right)\left(\zeta^{3}-\xi^{3}\right)\left(\xi^{3}-\eta^{3}\right) \\
& =-A \quad\left(\xi^{3}+\eta^{3}+\zeta^{3}\right)^{2} \\
& +\left(12 l^{2} A+4 l B\right) \quad\left(\xi^{3}+\eta^{3}+\zeta^{3}\right) \xi \eta \zeta \\
& +\left(18 l A+\left(1+8 l^{3}\right) B\right) \quad \xi^{2} \eta^{2} \zeta^{2} \\
& +\left(\left(4 l^{2}+8 l^{3}\right) A+4 l^{2} B\right)\left(\eta^{3} \zeta^{3}+\zeta^{3} \xi^{3}+\xi^{3} \eta^{3}\right) .
\end{aligned}
$$

The coefficient of $\eta^{3} \xi^{3}+\zeta^{3} \xi^{3}+\xi^{3} \eta^{3}$ on the right-hand side will vanish if $\left(1+2 l^{3}\right) A+l^{2} B=0$, or, what is the same thing, if $A=l^{2}, B=-\left(1+2 l^{3}\right)$; and substituting these values, we obtain

$$
\begin{aligned}
& \left\{l^{2}\left(x^{3}+y^{3}+z^{3}\right)-\left(1+2 l^{3}\right) \xi \eta \zeta\right\} \div\left(\eta^{3}-\zeta^{3}\right)\left(\zeta^{3}-\xi^{3}\right)\left(\xi^{3}-\eta^{3}\right) \\
& =-l^{2} \quad\left(\xi^{3}+\eta^{3}+\zeta^{3}\right) \\
& +\left(-4 l+4 l^{4}\right) \quad\left(\xi^{3}+\eta^{3}+\zeta^{3}\right) \xi \eta \zeta \\
& +\left(-1+8 l^{3}-16 l^{6}\right) \xi^{2} \eta^{2} \zeta^{2},
\end{aligned}
$$

or, what is the same thing,

$$
\begin{aligned}
l^{2}\left(x^{3}+y^{3}+z^{3}\right)-\left(1+2 l^{3}\right) x y z= & -\left(\eta^{3}-\zeta^{3}\right)\left(\zeta^{3}-\xi^{3}\right)\left(\xi^{3}-\eta^{3}\right) \\
& \times\left\{-l\left(\xi^{3}+\eta^{3}+\zeta^{3}\right)+\left(-1+4 l^{3}\right) \xi \eta \zeta\right\}^{2} .
\end{aligned}
$$

Hence the left-hand side vanishes in virtue of the relation between $\xi, \eta$, $\zeta$, or we have

$$
l^{2}\left(x^{3}+y^{3}+z^{3}\right)-\left(1+2 l^{3}\right) x y z=0,
$$

which proves the theorem.

36. Suppose that $(X, Y, Z)$ are the coordinates of a point of the Hessian, and let $(P, Q, R)$ be the coordinates of the point in which the tangent to the Hessian at the point $(X, Y, Z)$ again meets the Hessian, or, what is the same thing, the 
satellite point in regard to the Hessian of the tangent at $(X, Y, Z)$. And consider the conic

$$
X\left(x^{2}+2 l y z\right)+Y\left(y^{2}+2 l z x\right)+Z\left(x^{2}+2 l x y\right)
$$

which is the first or conic polar of the point $(X, Y, Z)$ in respect of the cubic. The polar (in respect to this conic) of the point $(P, Q, R)$ will be

$$
\xi x+\eta y+\zeta z=0
$$

where

$$
\begin{aligned}
& \xi=P X+l(R Y+Q Z) \\
& \eta=Q Y+l(P Z+R X) \\
& \zeta=R Z+l(Q X+P Y)
\end{aligned}
$$

or putting for $(P, Q, R)$ their values,

$$
\begin{aligned}
& \xi=\left(Y^{3}-Z^{3}\right)\left(X^{2}-l Y Z\right), \\
& \eta=\left(Z^{3}-X^{3}\right)\left(Y^{2}-l Z X\right), \\
& \zeta=\left(X^{3}-Y^{3}\right)\left(Z^{2}-l X Y\right)
\end{aligned}
$$

and if from these equations and the equation of the Hessian we eliminate $(X, Y, Z)$, we shall obtain the equation in line coordinates of the curve which is the envelope of the line $\xi x+\eta y+\zeta z=0$. We find, in fact,

$$
\begin{aligned}
& \xi^{3}+\eta^{3}+\zeta^{3}=\left(Y^{3}-Z^{3}\right)\left(Z^{3}-X^{3}\right)\left(X^{3}-Y^{3}\right)
\end{aligned}
$$

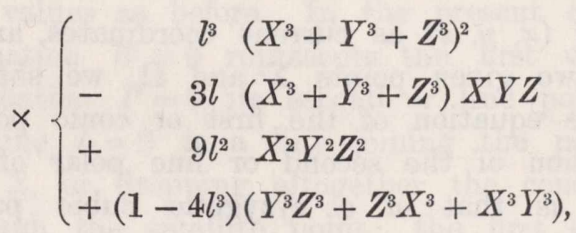

$$
\begin{aligned}
& \xi \eta \zeta=\left(Y^{3}-Z^{3}\right)\left(Z^{3}-X^{3}\right)\left(X^{3}-Y^{3}\right) \\
& \times\left\{\begin{array}{c}
l^{2}\left(X^{3}+Y^{3}+Z^{3}\right) X Y Z \\
+\left(1-l^{3}\right) X^{2} Y^{2} Z^{2} \\
-\quad l\left(Y^{3} Z^{3}+Z^{3} X^{3}+X^{3} Y^{3}\right)
\end{array}\right.
\end{aligned}
$$

and thence recollecting that

$$
H U=l^{2}\left(X^{3}+Y^{3}+Z^{3}\right)-\left(1+2 l^{3}\right) X Y Z,
$$

we find

$$
-l\left(\xi^{3}+\eta^{3}+\zeta^{3}\right)+\left(-1+4 l^{3}\right) \xi \eta \zeta=-\left(Y^{3}-Z^{3}\right)\left(Z^{3}-X^{3}\right)\left(X^{3}-Y^{3}\right)(H U)^{2},
$$

and the equation of the envelope is

$$
-l\left(\xi^{3}+\eta^{3}+\zeta^{3}\right)+\left(-1+4 l^{3}\right) \xi \eta \zeta=0,
$$

which is therefore the Pippian. We have thus the theorem: 
The envelope of the polar of the satellite point in respect to the Hessian of the tangent at any point of the Hessian, such polar being in respect of the conic which is the first or conic polar of the point of the Hessian in respect of the cubic, is the Pippian.

Article Nos. 37 to 40.-Investigations and theorems relating to the first or conic polar of a point of the cubic.

37. The investigations next following depend on the identical equations

$$
\begin{aligned}
& \left\{\alpha\left(X^{2}+2 l Y Z\right)+\beta\left(Y^{2}+2 l Z X\right)+\gamma\left(Z^{2}+2 l X Y\right)\right\} \\
& \quad \times\left\{-X Y Z\left(x^{3}+y^{3}+z^{3}\right)+\left(X^{3}+Y^{3}+Z^{3}\right) x y z\right\} \\
& =\left\{X\left(x^{2}+2 l y z\right)+Y\left(y^{2}+2 l z x\right)+Z\left(z^{2}+2 l x y\right)\right\} \\
& \quad \times\left\{X\left(Y^{3}-Z^{3}\right)(\gamma y-\beta z)+Y\left(Z^{3}-X^{3}\right)(\alpha z-\gamma x)+Z\left(X^{3}-Y^{3}\right)(\beta x-\alpha y)\right\} \\
& +\left\{x\left(X^{2}+2 l Y Z\right)+y\left(Y^{2}+2 l Z X\right)+z\left(Z^{2}+2 l X Y\right)\right\} \\
& \quad \times\left\{-(\alpha Y Z+\beta Z X+\gamma X Y)\left(X x^{2}+Y y^{2}+Z z^{2}\right)+\left(\alpha X^{2}+\beta Y^{2}+\gamma Z^{2}\right)(X Y z+Y z x+Z x y)\right\},
\end{aligned}
$$

which is easily verified.

I represent the equation in question by

$$
K \Upsilon=W L+P \Theta ;
$$

then considering $(x, y, z)$ as current coordinates, and $(X, Y, Z)$ and $(\alpha, \beta, \gamma)$ as the coordinates of two given points $\Sigma$ and $\Omega$, we shall have $U=0$ the equation of the cubic, $W=0$ the equation of the first or conic polar of $\Sigma$ with respect to the cubic, $P=0$ the equation of the second or line polar of $\Sigma$ with respect to the cubic. The equation $\Upsilon=0$ is that of a syzygetic cubic passing through the point $\Sigma$ : the coordinates of the satellite point in respect to this syzygetic cubic of its tangent at $\Sigma$ are

$$
X\left(Y^{3}-Z^{3}\right): Y\left(Z^{3}-X^{3}\right): Z\left(X^{3}-Y^{3}\right) ;
$$

and calling the point in question $\Sigma^{\prime}$, then $L=0$ is the equation of a line through the points $\Sigma^{\prime}, \Omega$. The equation $\Theta=0$ is that of a conic, viz. the first or conic polar of $\Sigma$ with respect to a certain syzygetic cubic

$$
-2(\alpha Y Z+\beta Z X+\gamma X Y)\left(x^{3}+y^{3}+z^{3}\right)+\left(\alpha X^{2}+\beta Y^{2}+\gamma Z^{2}\right) x y z=0,
$$

depending on the points $\Sigma, \Omega$, or, what is the same thing, the conic $\Theta=0$ is a properly selected conic passing through the points of intersection of the first or conic polars of $\Sigma$ with respect to any two syzygetic cubics; and lastly, $K$ is a constant coefficient. The equation expresses that the points of intersection of

$$
(W=0, P=0),(W=0, \Theta=0),(L=0, P=0),(L=0, \Theta=0),
$$

lie in the syzygetic cubic $\Upsilon=0$. 
The left-hand side of the equation may be written

$$
\begin{aligned}
& -X Y Z\left\{\alpha\left(X^{2}+2 l Y Z\right)+\beta\left(Y^{2}+2 l Z X\right)+\gamma\left(Z^{2}+2 l X Y\right)\right\}\left(x^{3}+y^{3}+z^{3}+6 l x y z\right) \\
& +\quad x y z\left\{\alpha\left(X^{2}+2 l Y Z\right)+\beta\left(Y^{2}+2 l Z X\right)+\gamma\left(Z^{2}+2 l X Y\right)\right\}\left(X^{3}+Y^{3}+Z^{3}+6 l X Y Z\right)
\end{aligned}
$$

and it may be remarked also that we have

$$
-3 X Y Z\left\{\alpha\left(X^{2}+2 l Y Z\right)+\beta\left(Y^{2}+2 l Z X\right)+\gamma\left(Z^{2}+2 l X Y\right)\right\}
$$

equal identically to

$$
\begin{array}{r}
\left\{X\left(Y^{3}-Z^{3}\right)(\gamma Y-\beta Z)+Y\left(Z^{3}-X^{3}\right)(\alpha Z-\gamma X)+Z\left(X^{3}-Y^{3}\right)(\beta X-\alpha Y)\right\} \\
-(\alpha Y Z+\beta Z X+\gamma X Y)\left(X^{3}+Y^{3}+Z^{3}+6 l X Y Z\right) .
\end{array}
$$

Hence if we assume

$$
X^{3}+Y^{3}+Z^{3}+6 l X Y Z=0,
$$

the equation will take the form

$$
K U=W L+P \Theta,
$$

where the constant coefficient $K$ may be expressed under the two different forms

$$
\begin{aligned}
K & =-X Y Z\left\{\alpha\left(X^{2}+2 l Y Z\right)+\beta\left(Y^{2}+2 l Z X\right)+\gamma\left(Z^{2}+2 l X Y\right)\right\} \\
& =\frac{1}{3}\left\{X\left(Y^{3}-Z^{3}\right)(\gamma Y-\beta Z)+Y\left(Z^{3}-X^{3}\right)(\alpha Z-\gamma X)+Z\left(X^{3}-Y^{3}\right)(\beta X-\alpha Y)\right\},
\end{aligned}
$$

and $W, L, P, \Theta$ have the same values as before. In the present case the point $\Sigma$ is a point of the cubic: the equation $W=0$ represents the first or conic polar of the point in question, and the equation $P=0$ its second or line polar, which is also the tangent of the cubic. The line $L=0$ is a line joining the point $\Omega$ with the satellite point of the tangent at $\Sigma$, or dropping altogether the consideration of the point $\Omega$, is an arbitrary line through the satellite point: the first or conic polar of $\Sigma$ meets the cubic twice in the point $\Sigma$, and therefore also meets it in four other points; the conic $\Theta=0$ is a conic passing through these four points, and completely determined when the particular position of the line through the satellite point is given. And, as before remarked, $\Theta=0$ is a conic passing through the points of intersection of the first or conic polars of $\Sigma$ with respect to any two syzygetic cubics. We have thus the theorem:

The first or conic polar of a point of the cubic touches the cubic at this point, and besides meets it in four other points; the four points in question are the points in which the first or conic polar of the given point in respect of the cubic is intersected by the first or conic polar of the same point in respect to any syzygetic cubic whatever.

38. The analytical result may be thus stated: putting

$$
\kappa=\alpha Y Z+\beta Z X+\gamma X Y, \quad \lambda=\alpha X^{2}+\beta Y^{2}+\gamma Z^{2},
$$


or, if we please, considering $\kappa, \lambda$ as arbitrary parameters, then the four points lie in the conic

$$
(2 \kappa X, 2 \kappa Y, 2 \kappa Z,-\lambda X,-\lambda Y,-\lambda Z \gamma x, y, z)^{2}=0,
$$

or, what is the same thing, they are the points of intersection of the two conics

$$
\begin{aligned}
& X x^{2}+Y y^{2}+Z z^{2}=0 \\
& X y z+Y z x+Z x y=0
\end{aligned}
$$

39. Considering the four points as the angles of a quadrangle, it may be shown that the three centres of the quadrangle lie on the cubic. To effect this, assume that the conic

$$
(2 \kappa X, 2 \kappa Y, 2 \kappa Z,-\lambda X,-\lambda Y,-\lambda Z \gamma x, y, z)^{2}=0
$$

represents a pair of lines; these lines will intersect in a point, which is one of the three centres in question. And taking $x, y, z$ as the coordinates of this point, we have

$$
\begin{aligned}
x^{2}: y^{2}: z^{2}: y z: z x: x y & =4 \kappa^{2} Y Z-\lambda^{2} X^{2} \\
& : 4 \kappa^{2} Z X-\lambda^{2} Y^{2} \\
& : 4 \kappa^{2} X Y-\lambda^{2} Z^{2} \\
: & \lambda^{2} Y Z+2 \kappa \lambda X^{2} \\
: & \lambda^{2} Z X+2 \kappa \lambda Y^{2} \\
: & \lambda^{2} X Y+2 \kappa \lambda Z^{2} ;
\end{aligned}
$$

and we may, if we please, use these equations to find the relation between $\kappa, \lambda$. Thus in the identical equation $x^{2} \cdot y^{2}-(x y)^{2}=0$, substituting for $x^{2}, x y, y^{2}$ their values, and throwing out the factor $Z$, we find $\left(4 \kappa^{3}-\lambda^{3}\right) X Y Z-\kappa \lambda^{2}\left(X^{3}+Y^{3}+Z^{3}\right)=0$, and thence, in virtue of the equation $X^{3}+Y^{3}+Z^{3}+6 l X Y Z=0$, we obtain

$$
4 \kappa^{3}+6 l \kappa \lambda^{2}-\lambda^{3}=0 \text {. }
$$

But the preceding system gives conversely,

$$
\begin{aligned}
X^{2}: Y^{2}: Z^{2}: Y Z: Z X: X Y & =4 \kappa^{2} y z-\lambda^{2} x^{2} \\
& : 4 \kappa^{2} z x-\lambda^{2} y^{2} \\
& : 4 \kappa^{2} x y-\lambda^{2} z^{2} \\
& : \lambda^{2} y z+2 \kappa \lambda x^{2} \\
& : \lambda^{2} z x+2 \kappa \lambda y^{2} \\
& : \lambda^{2} x y+2 \kappa \lambda z^{2} .
\end{aligned}
$$

Hence from the identical relation $X^{2} \cdot Y^{2}-(X Y)^{2}=0$, substituting for $X^{2}, X Y, Y^{2}$ their values, and throwing out the factor $z$, we find $\left(4 \kappa^{3}-\lambda^{3}\right) x y z-\kappa \lambda^{2}\left(x^{3}+y^{3}+z^{3}\right)=0$, and thence, in virtue of the equation $4 \kappa^{3}-\lambda^{3}=-6 l \kappa \lambda^{2}$, we obtain

$$
x^{3}+y^{3}+z^{3}+6 l x y z=0,
$$


which shows that the point in question lies on the cubic. We have thus the theorem :

The first or conic polar of a point of the cubic touches the cubic at the point, and meets it besides in four points, which are the angles of a quadrangle the centres of which lie on the cubic. In other words, the quadrangle is an inscribed quadrangle.

40. To find the equations of the three axes of the quadrangle, that is of the lines through two centres.

We have

$$
\begin{aligned}
& \left(4 \kappa^{2} Y Z-\lambda^{2} X^{2}\right) x+\left(\lambda^{2} X Y+2 \kappa \lambda Z^{2}\right) y+\left(\lambda^{2} Z X+2 \kappa \lambda Y^{2}\right) z=0 \\
& \left(\lambda^{2} X Y+2 \kappa \lambda Z^{2}\right) x+\left(4 \kappa^{2} Z X-\lambda^{2} Y^{2}\right) y+\left(\lambda^{2} Y Z+2 \kappa \lambda X^{2}\right) z=0 \\
& \left(\lambda^{2} Z X+2 \kappa \lambda Y^{2}\right) x+\left(\lambda^{2} Y Z+2 \kappa \lambda X^{2}\right) y+\left(4 \kappa^{2} X Y-\lambda^{2} Z^{2}\right) z=0
\end{aligned}
$$

or arranging these equations in the proper form and eliminating $\kappa^{2}, \kappa \lambda, \lambda^{2}$, we find

$$
\begin{array}{lll}
Y Z x, & Z^{2} y+Y^{2} z, & X(-X x+Y y+Z z) \\
Z X y, & X^{2} z+Z^{2} x, & Y(X x-Y y+Z z) \\
X Y z, & Y^{2} x+X^{2} y, & Z(X x+Y y+Z z)
\end{array} \mid=r
$$

or, multiplying out,

$$
\begin{aligned}
& X Y Z\left\{\left(Z^{3}-Y^{3}\right) x^{3}+\left(X^{3}-Z^{3}\right) y^{3}+\left(Y^{3}-X^{3}\right) z^{3}\right\} \\
&+x^{2} y Z Y^{2}\left(-2 X^{3}+Y^{3}+Z^{3}\right)+z x^{2} Y Z^{2}\left(2 X^{3}-Y^{3}-Z^{3}\right) \\
&+y^{2} z X Z^{2}\left(-2 Y^{3}+Z^{3}+X^{3}\right)+x y^{2} Z X^{2}\left(2 Y^{3}-Z^{3}-X^{3}\right) \\
&+z^{2} x Y X^{2}\left(-2 Z^{3}+X^{3}+Y^{3}\right)+y z^{2} X Y^{2}\left(2 Z^{3}-X^{3}-Y^{3}\right)=0 .
\end{aligned}
$$

We may simplify this result by means of the equation $X^{3}+Y^{3}+Z^{3}+6 l X Y Z=0$, so as to make the left-hand side divide out by $X Y Z$ : we thus obtain

$$
\begin{aligned}
& \left(Z^{3}-Y^{3}\right) x^{3}+\left(X^{3}-Z^{3}\right) y^{3}+\left(Y^{3}-X^{3}\right) z^{3} \\
+ & \left(-3 X^{2} Y-6 l Y^{2} Z\right) x^{2} y+\left(-3 Y^{2} Z-6 l Z^{2} X\right) y^{2} z+\left(-3 Z^{2} X-6 l X^{2} Y\right) z^{2} x \\
+ & \left(3 X Y^{2}+6 l X^{2} Z\right) x y^{2}+\left(3 Y Z^{2}+6 l Y^{2} X\right) y z^{2}+\left(3 Z X^{2}+6 l Z^{2} Y\right) z x^{2}=0
\end{aligned}
$$

or in a different form,

$$
\begin{aligned}
& \left(y^{3}-z^{3}\right) X^{3}+\left(z^{3}-x^{3}\right) Y^{3}+\left(x^{3}-y^{3}\right) Z^{3} \\
+ & \left(-3 x^{2} y-6 l z^{2} x\right) X^{2} Y+\left(-3 y^{2} z-6 l x^{2} y\right) Y^{2} Z+\left(-3 z^{2} x-6 l y^{2} z\right) Z^{2} X \\
+ & \left(3 x y^{2}+6 l y z^{2}\right) X Y^{2}+\left(3 y z^{2}+6 l z x^{2}\right) Y Z^{2}+\left(3 z x^{2}+6 l x y^{2}\right) Z X^{2}=0
\end{aligned}
$$

as the equation of the three axes of the quadrangle. 


\section{Article No. 41. Recapitulation of geometrical definitions of the Pippian.}

In conclusion, I will recapitulate the different modes of generation or geometrical definitions of the Pippian, obtained in the course of the present memoir. The curve in question is:

1. The envelope of the line joining a pair of conjugate poles of the cubic (see Nos. 2 and 13).

2. The envelope of each line of the pair forming the first or conic polar with respect to the cubic of a conjugate pole of the cubic (see Nos. 2 and 14).

3. The envelope of a line which is the polar of a conjugate pole of the cubic, with respect to the conic which is the first or conic polar of the other conjugate pole in respect to any syzygetic cubic (see Nos. 2 and 9).

4. The locus of the harmonic with respect to a pair of conjugate poles of the cubic of the third point of intersection with the Hessian of the line joining the two conjugate poles (see Nos. 2 and 17).

5. The envelope of a line such that its lineo-polar envelope with respect to the cubic breaks up into a pair of lines (see No. 24).

6. The envelope of a line which meets three conics, the first or conic polars of any three points in respect to the cubic, in six points in involution (see No. 22).

7. The envelope of the second or line polar with respect to the cubic, of a point the locus of which is a certain curve of the sixth order in quadratic syzygy with the cubic and Hessian, viz. the curve $-S . U^{2}+(H U)^{2}=0$ (see No. 27).

8. The envelope of a line having for its satellite point a point of the Hessian (see No. 35).

9. The envelope of the polar of the satellite point with respect to the Hessian of the tangent at a point of the Hessian, with respect to the first or conic polar of the point of the Hessian in respect to the cubic (see No. 36). 